(C) Academy of Science of South Africa

February 2018

\section{ISBN 978-0-9947117-5-5}

DOI http://dx.doi.org/10.17159/assaf.2018/0020

Published by:

Academy of Science of South Africa (ASSAf)

PO Box 72135, Lynnwood Ridge, Pretoria, South Africa, 0040

Tel: +27 123496600 • Fax: +27865769520

E-mail:admin@assaf.org.za

Reproduction is permitted, provided the source and publisher are appropriately acknowledged.

The Academy of Science of South Africa (ASSAf) was inaugurated in May 1996. It was formed in response to the need for an Academy of Science consonant with the dawn of democracy in South Africa: activist in its mission of using science and scholarship for the benefit of society, with a mandate encompassing all scholarly disciplines that use an open-minded and evidence-based approach to build knowledge. ASSAf thus adopted in its name the term 'science' in the singular as reflecting a common way of enquiring rather than an aggregation of different disciplines. Its Members are elected on the basis of a combination of two principal criteria, academic excellence and significant contributions to society.

The Parliament of South Africa passed the Academy of Science of South Africa Act (No 67 of 2001), which came into force on 15 May 2002. This made ASSAf the only academy of science in South Africa officially recognised by government and representing the country in the international community of science academies and elsewhere.

This report reflects the proceedings of the GenderilnSITE Elsevier Foundation Thematic Workshop - Gender and Innovation: Implications for Sustainable Development held on 4 - 6 September 2017 at the Technology Innovation Agency, TIA House, Pretoria, South Africa. Views expressed are those of the individuals and not necessarily those of the Academy nor a consensus view of the Academy based on an in-depth evidence-based study. 


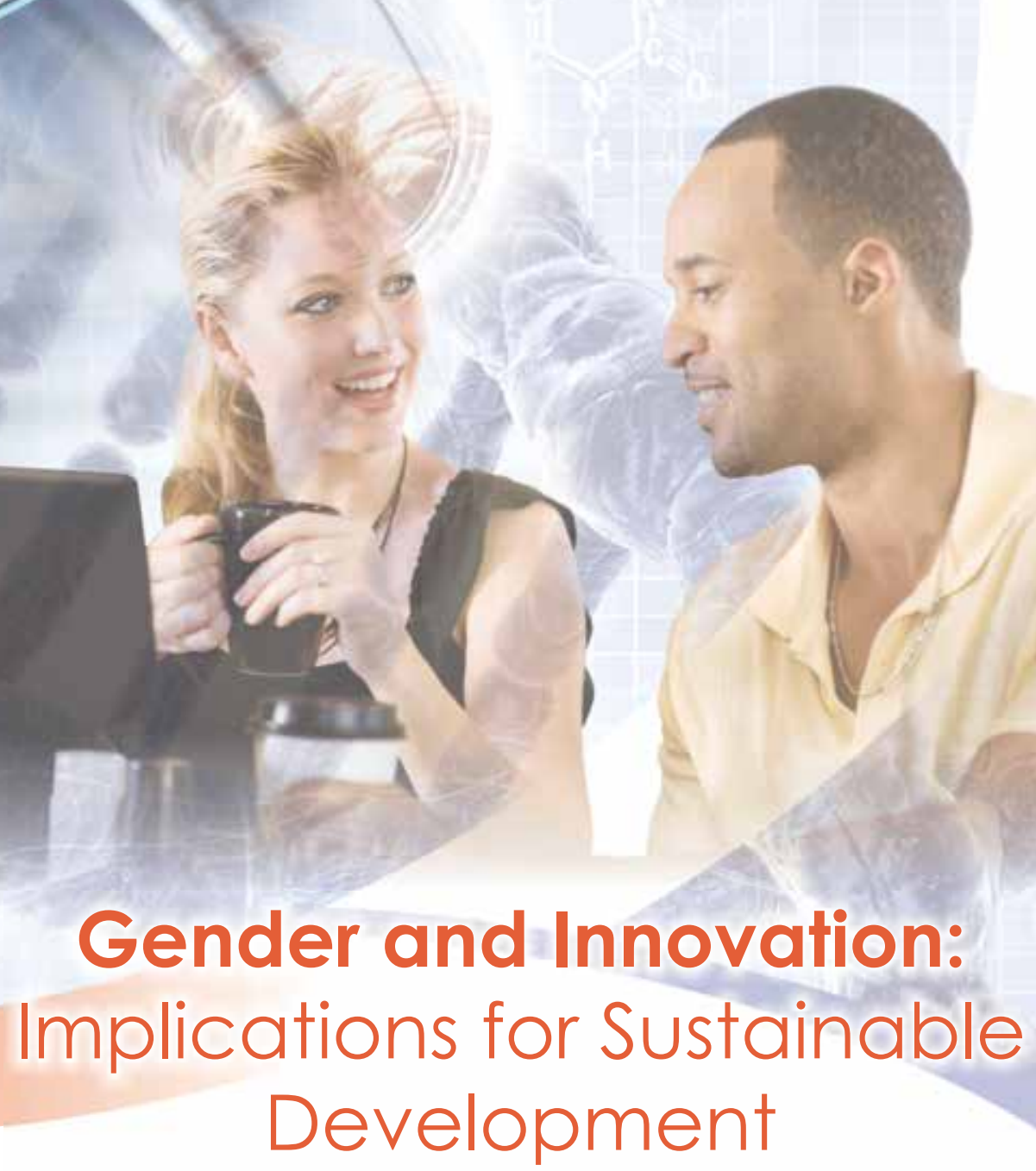

GenderInSITE Elsevier Foundation Thematic Workshop 


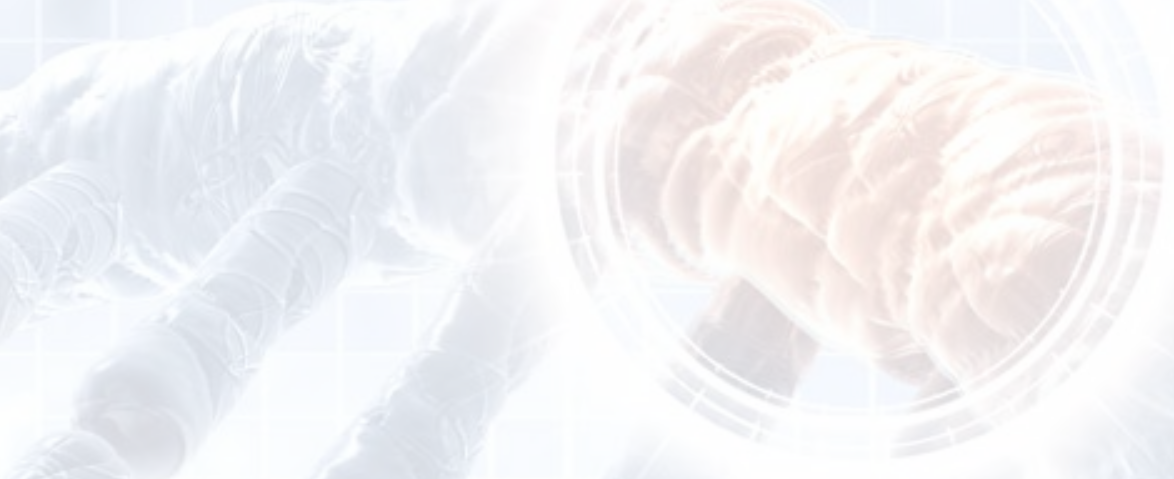




\section{TABLE OF CONTENTS}

DAY 1 (MODERATOR: ROSEANNE DIAB)

WELCOME AND INTRODUCTIONS

Roseanne Diab, Executive Officer, Academy of Science of South Africa (ASSAf): Regional Focal Point for Southern Africa of Gender in Science, Innovation, Technology and Engineering (GenderlnSITE)

Iris Kisjes, Senior External Relations Manager,

Elsevier Foundation

Alice Abreu, Director, GenderInSITE, Federal University of Rio de Janeiro, Brazil

Patrick Krappie, Corporate Relations and Strategic

Engagements, Technology Innovation Agency (TIA)

Imraan Patel, Deputy Director-General, Socio-Economic Innovation Partnerships, Department of Science and Technology (DST), South Africa

\section{AN INTRODUCTION TO GENDERINSITE}

GenderlnSITE: Transforming the Policy Landscape (Shirley Malcom, GenderlnSITE Co-Chair, Director of Education and Human Resources Programmes, American Association for the Advancement of Science (AAAS))

KEYNOTE ADDRESS

Gendered Innovations in Science, Health and Medicine, Engineering, and Environment (Londa Schiebinger, the John L Hinds Professor of History of Science, Director, Gendered Innovations in Science, Health and Medicine, Engineering and Environment, Stanford University)

QUESTIONS AND COMMENTS

PANEL 1

Gendered Research and Inclusive Innovation in the University Strategy (Simone Buitendijk, Vice-Provost (Education), Imperial College, United Kingdom (UK)) 
Applying a Gender Lens to Science, Technology and Innovation (Dong Wu, Chief, Policy Review Section, Officer-inCharge, Science and Technology Section, United Nations (UN) Conference on Trade and Development (UNCTAD))

Impediments to the Full Participation of Women in Innovation (Jennifer Thomson, GenderlnSITE Co-Chair, President, Organisation for Women in Science for the Developing World (OWSD); University of Cape Town, South Africa)

Creating Improvements in Innovation for Women (Sharifah Maimunah Syed Zin, Director, International Science, Technology and Innovation Centre for South South Co-operation (ISTIC))

PANEL 2

Innovation Beyond Stirring: Reflecting Dimensions of Gender in what Works (Nick Perkins, Lead Technical Advisor: Development Communication and Extension, Centre for Agriculture and Biosciences International (CABI))

Gender and Innovation: An Asian Perspective (Miyoko Watanabe, Deputy Executive Director, Director-General of Centre for Science Communication and Director of Office for Diversity and Inclusion, Japan Science and Technology Agency)

Gender, Science, Technology and Innovation (STI) and the Sustainable Development Goals (SDGs) - South African Developments (Imraan Patel, Deputy Director-General, SocioEconomic Innovation Partnerships, DST)

\section{QUESTIONS AND COMMENTS}

PANEL 3

Gender and Research, New Results and Activities in Germany (Martina Schraudner, Head, Fraunhofer Centre for Responsible Research and Innovation (CeRRI) at the Institute for Industrial Engineering (IAO), and Chair for Gender and Diversity in Technology and Product Development at the Technical University, Berlin (via Skype))

Gender and Innovation at São Paulo Research Foundation (FAPESP) (Ana Maria Fonseca de Almeida, Assistant Coordinator of Social and Human Sciences, FAPESP) 
Promoting Women Entrepreneurship Development via the Inclusion of Technology and Innovation (Jill Sawers, Director: FemTech (Pty) Ltd)

Women in Technology Businesses - Accessibility to Funding, Support and Markets: TIA Case Studies (Anitha Ramsuran, Manager: Innovation for Inclusive Development, TIA)

\section{QUESTIONS AND COMMENTS}

PANEL 4

Measuring Women's Participation in International Patenting (Bruno Le Feuvre, Statistical Analyst, IP Statistics Section, World Intellectual Property Organisation Geneva 20 (WIPO) (via Skype))

A Newe Way by Her Invented (Amy Everard, 1637)

(Anne Grobler, Director: DST/North-West University (NWU)

Preclinical Platform, NWU)

Contribution to Greater Equity in the Attainment of the SDGs (Berenice Marais, Executive, Innovation Enabling and Support Programmes, TIA)

\section{QUESTIONS AND COMMENTS}

DAY 2 (MODERATOR: ALICE ABREU)

PANEL 5

Gendered Innovations (Anne Githuku-Shongwe,

Representative for East and South African Region,

UN Women)

The Transition from Research/Invention to Innovation - Is it Gender Neutral? (Neville Comins, Independent Consultant)

Gender and Entrepreneurship - Potential Benefits for Investment in Innovations (Chamunorwa Togo, General Manager: Innovation and Industry Development, The Innovation Hub Management Company)

Gender and Scientific Research: Perspectives from a Gender Audit of Kenya's Climate Change Policies (Winnie Khaemba, Research Fellow, African Centre for Technology Studies (ACTS)) 
REPORT TO PLENARY (MODERATOR: IRIS KISJES)

Group 1: From Research to Applications: Gendered Innovations and Other Challenges (Neville Comins)

Group 2: Scientific Research and SITE Education

(Shirley Malcom)

Group 3: Gender Equity in the Attainment of the SDGs:

Influencing Policy (Alice Abreu)

QUESTIONS AND COMMENTS

DAY 3 (MODERATOR: ALICE ABREU)__ 73

GENERAL DISCUSSION

Structure of the Report (the main issues and how they

fit together)

Clarification of the Purpose of the Case Study Project 73

Comments

74

Projects

Other Points Raised about the Projects $\quad 79$

From Motivation to Implementation $\quad 79$

Action Items $\quad 80$

MAIN CONCLUSIONS AND RECOMMENDATIONS 80

Other $\quad 81$

WRAP-UP, CLOSING AND THANKS 82

ANNEXURE A: LIST OF ACRONYMS___ 83

APPENDIX B: LIST OF PARTICIPANTS _ 85 


\section{DAY 1}

(MODERATOR: PROF ROSEANNE DIAB)

\section{WELCOME AND INTRODUCTIONS}

\section{Prof Roseanne Diab, Executive Officer, Academy of Science of South Africa (ASSAf): Regional Focal Point for Southern Africa of Gender in Science, Innovation, Technology and Engineering (GenderInSITE)}

Prof Diab extended a warm welcome to everyone, particularly the international visitors, on behalf of ASSAf and the team that organised the workshop, as well as the GenderlnSITE Focal Point for Southern Africa. The event was the second in a series organised under the auspices of GenderInSITE and the Elsevier Foundation. Innovation is a topic that, for the Academy and the GenderlnSITE Focal Point, was under-explored. She thanked the Technology Innovation Agency (TIA) for hosting the workshop and the sponsors for their support.

It was anticipated that this small, select group attending the workshop would have robust and engaging discussions. Outputs from the workshop would include a proceedings report giving a record of all the presentations and discussions that took place, a short policymakers brief that would identify key findings and recommendations, and action items that would be identified and taken up by the GenderlnSITE network.

\section{Ms Iris Kisjes, Senior External Relations Manager, Elsevier Foundation}

The Elsevier Foundation was proud to be able to play a central role in supporting GenderlnSITE, especially the workshops in Argentina and South Africa. Elsevier Director, Ylann Schemm, regretted being unable to attend the workshop.

Ms Kisjes is part of the board for the Elsevier Women's Network and helped implement Economic Dividends for Gender Equality (EDGE), for which Elsevier had achieved certification. She is also responsible for the Elsevier's Sustainable Development Goals (SDGs) reports, such as the Sustainability Science in the Global Research Landscape showcasing benchmarks on progress made in respect of the SDGs. She is involved in the Organisation for Women in Science for the Developing World (OWSD) through the Elsevier Foundation Awards for Young Women in Science, which is an inspiring experience.

The Elsevier Foundation is also involved in the Gender Summits that take place every year and the Science, Technology and Medical Journals 
(STMJ) gender working group, making sure that there are industry standards on editorial policies on sex and gender, and gender diversity of reviewers, panellists, editors and board members.

The Elsevier Foundation Board meets this week to discuss changes to the Foundation in order for it to contribute better to critical issues in science, health and development. The Foundation has been focusing on access to information and the building of skills but the role of technology has become increasingly important in these areas and information analytics is central to shaping change and responding to the SDGs.

The Foundation intends to double its investment in technology-oriented health and science programmes and its goal is to identify, develop and fund projects that enable data scientists to find solutions to these global challenges, and to scale these up over time for wider impact. Ms Kisjes invited participants to consider ideas about how this could be incorporated into its current programmes.

Several pilot projects through partnerships with DataKind and Amref Health Africa started this year. The board will discuss which technologies and solutions offer the best opportunities to advance global health and development in the coming years and how technology can be embedded into existing partnerships, and consider whether the matter of diversity and inclusion in the science and health sectors should continue to be an area of focus for the Foundation.

\section{Prof Alice Abreu, Director GenderInSITE, Federal University of Rio de Janeiro, Brazil}

Innovation was always in the deoxyribonucleic acid (DNA) of GenderlnSITE, but this is the first significant activity related to innovation and would not have happened without the help of many persons and institutions, which were thanked.

The idea of having a high-level discussion with international specialists on the theme of gendered innovation was always sustained by Geoffrey Oldham, member of GenderInSITE since its creation and who, together with Shirley Malcom, has a long history of involvement with gender in science since the day of the Gender Working Group at the United Nations (UN) Commission for Science and Technology (S\&T) for Development. His vision and clear focus on the need to explore the implications for the developing world of the work done in the North and the need to have a clear research agenda for gender and innovation 
to support the effective implementation of the SDGs was the initial motivation for this meeting, and very quickly got the full support of the GenderInSITE team. Prof Oldham' was not able to be at this workshop due to health issues, but he would be proud of the result of our efforts.

Prof Abreu thanked Ylann Schemm, Director of the Elsevier Foundation and long-time supporter of GenderlnSITE. The Elsevier Foundation has provided the essential support for two central GenderInSITE activities: this workshop and the previous workshop on the integration of gender perspectives in S\&T in higher education held in Buenos Aires last April, under the co-ordination of the GenderInSITE Latin American Regional Focal Point.

All of this would not have materialised into this workshop without the hard work of Prof Diab and her team. Heartfelt thanks to the whole ASSAf team that has brought this event to fruition. It is due to their impressive work that the valuable local support of TIA, the hosting institution, and the Department of Science and Technology (DST) was garnered. GenderlnSITE is grateful for their sponsorship and hopeful that this workshop will bring an important contribution to their work on innovation for development.

\section{Prof Abreu acknowledged:}

- GenderlnSITE Co-Chairs, Shirley Malcom and Jennifer Thomson, who supported this idea from the onset and continued to bring their valuable contributions to this discussion.

- Erin Johnson, whose work in GenderInSITE has sustained its activities both in the real world and in the worldwide web with the impressive webpage she has managed in the recent years.

- The participants of this workshop, who will help to take the discussion on this important theme forward.

Being able to bring together such high-level specialists and friends is the central achievement of this event and has influenced the way this workshop is organised, making sure that everyone will be heard and fully integrate the contribution that each one brings to the table. It was necessary to break the discussions into smaller groups in order to be able to distil all the information into clear findings and recommendations. The breakout sessions aim to:

Geoff Oldham passed away soon after this workshop was held. He is remembered for his immense contribution to the work of GenderInSITE and in particular for his support in the early planning stages of this workshop. 
- Filter the discussion into the central idea of this meeting, which is to understand and develop the intricate road from science to innovation and explore the differential impact that innovation has on the lives of men and women, especially the new technologies introduced in development programmes.

- Understand what will be needed in terms of scientific research in SITE and SITE education to support a real development of gendered innovation.

- Discuss how to help bridge the gap between knowledge of SITE and gender issues, and those who need to access this knowledge for policy and decision-making, i.e. to identify the knowledge brokers and highlight the importance of this for the effective implementation of the SDGs.

It is hoped that the focused discussion will come up with significant recommendations to policymakers, as well as an agenda of action items to achieve goals and targets that would effectively lead to gender-aware innovation and technology, especially from the view of the South, which is still lacking.

\section{Mr Patrick Krappie, Corporate Relations and Strategic Engagements, TIA}

Mr Krappie welcomed all workshop participants to the TIA on behalf of the Chief Executive Officer (CEO), Mr Barlow Manilal.

TIA embraced the opportunity to be the hosting partner of the GenderlnSITE workshop, which was important to TIA, particularly in the context of the current discourse around the knowledge economy and innovation and their contribution to economic development and global competitiveness of South African industries. Policymakers in the developing world have a particular interest in innovation. The discussions on gendered innovation are relevant to a number of imperatives of developmental states. They require that consideration be given to what it means to specific sectors of society and that there be recognition for the broader concept of innovation for inclusive development that serves a wide range of stakeholders and addresses a number of issues.

TIA concerns itself with the youth, the poor, the disabled, as well as gender equity. As an agency of the DST, TIA is expected to respond to challenges of these sectors of society and women as it executes its mandate. The DST's corporate strategy has specific intentions to target skills development and empowerment of women in science, 
technology and innovation (STI). During the 2016 Science Forum hosted by the DST, TIA coordinated a thematic session on women in innovation that discussed ways to promote the role of women and enhance their participation in innovation and types of innovations that address women's challenges. TIA supports a number of companies that have developed solutions in this regard.

Mr Krappie wished the participants well with their deliberations.

\section{Mr Imraan Patel, Deputy Director-General, Socio-Economic Innovation Partnerships, DST, South Africa}

Mr Patel welcomed the guests from other parts of South Africa and abroad who represented an enviable collection of thought leaders in the area of gender and innovation.

One of the key objectives of the DST is to look at the National System of Innovation (NSI) that deals with exclusion of all types. Key to this exclusion is the issue of women and gender. South Africa's National Development Plan (NDP) identifies a triple challenge of unemployment, poverty and inequality. All of these have a strong gender component and must be dealt with at a governmental level. Although this is the start of a long journey, there are some important seeds of development that will grow towards an environment that has a much stronger gendersensitive innovation system.

This workshop is timely because it is part of the ongoing implementation of the NDP where the STI system needs to think about its own response. The DST has spent the last 18 months revising the White Paper on S\&T. Twenty years ago, the set of conditions differed considerably as the first democratic government had just come into being and it was necessary to deal with different challenges and to stabilise and re-orientate the NSI to be focused on the majority. Over time, it has become necessary to consider the implications in the context of the SDGs and the NDP to ensure a more inclusive next phase of human development.

The DST is looking forward to the outcome of the discussions of this workshop, particularly as the White Paper process is not yet finalised and opens up the space to consider what needs to be done practically to enhance and engender a stronger NSI. As part of the process, the DST will be reflecting deeply on its next Decadal Plan for STI in South Africa, which will hopefully unfold in the course of 2018. The DST was committed to championing the recommendations that would come out of this workshop that could be used for policy experimentation in its Decadal Plan. 
Mr Patel looked forward to follow-up discussions with ASSAf about the various recommendations of this workshop for the global community of influential players, as well as South Africa to build on what is already being done. He suggested that GenderlnSITE should be invited to reflect on progress made with regard to these recommendations in a few years' time.

\section{AN INTRODUCTION TO GENDERINSITE}

\section{GenderlnSITE: Transforming the Policy Landscape (Prof Shirley Malcom, GenderInSITE Co-Chair, Director of Education and Human Resources Programmes, American Association for the Advancement of Science (AAAS))}

GenderInSITE is based on the idea that STI for development policy and programmes will be more effective, equitable and sustainable when the gender lens is applied, and when policies and programmes reflect the visions, aims, concerns, perspectives, knowledge and abilities of both men and women.

GenderInSITE lives at the intersection of STI, development and gender and was conceptualised some years back. The process of looking at the gender dimensions that related to empowering women was undertaken and the ideas of transformative actions were introduced when the UN Commission on Science and Technology for Development (CSTD) began its work to support the Women's Conference in Beijing in 1995. When the symposium at the 1999 World Conference on Science in Budapest was held, the idea of gender as being important to the S\&T structure and to the discussion on development was introduced. Initially, people had to be convinced that S\&T was important to development; then they had to be convinced that there were gender concerns within STI and that S\&T needed to be introduced into the women's discussion, not only in world conferences related to science, but also at the Commission on the Status of Women ( $55^{\text {th }}$ Session) when empowerment of women was addressed.

GenderInSITE was actually born in a meeting room at the UN Educational, Scientific and Cultural Organisation (UNESCO). At that time representatives from CSTD, UNESCO, The World Academy of Sciences (TWAS) and OWSD were discussing how to move forward with the ambitious agenda of trying to operate at the intersection of gender, STI and development, and how to begin to influence the policy landscape in that space. When the idea of GenderlnSITE emerged, advantage was taken of the homophone of 'insight' (being able to 
see beyond) at the same time as talking about 'InSITE' as offering the dimension of STI and engineering. The group thought about what it wanted to see accomplished and what InSITE through a gender lens would bring. It was necessary to be intentional with regard to the intersectionalities that live within gender as well as race, ethnicity, geography and other aspects that are crucial to being able to see the way forward. It was hoped that this would promote a different way of thinking about the research, the agenda-setting, the processes, the policies and the actions that are crucial to solving some of the most challenging problems, such as those embedded in and articulated by the SDGs.

A quote supposedly by Einstein states that we cannot solve our problems with the same kind of thinking that created them. Much of the structure within science and engineering has been dictated by the most influential members of our communities via the current organisations and processes established to consider these. GenderlnSITE was established to renegotiate the contract between the public and the STI communities and as a reminder to view the different publics in many different ways. As with any living system, there must be adaptation to changing circumstances as things do not stay fixed. There must be responsiveness to the changes as they present themselves, and the policy dynamic is absolutely critical. This workshop offers the opportunity to start having this discussion around this critical component of innovation.

\section{KEYNOTE ADDRESS}

Gendered Innovations in Science, Health and Medicine, Engineering, and Environment (Prof Londa Schiebinger, the John L Hinds Professor of History of Science, Director, Gendered Innovations in Science, Health and Medicine, Engineering, and Environment, Stanford University)

Gendered Innovations is a project that was produced through a large international collaboration involving 80 basic (natural) scientists, engineers and gender experts through funding from the European Commission, the National Science Foundation and Stanford University. The project brought together scientists, engineers and gender experts, in many cases for the first time, and has taken off over the last few years. There are new policies in the European Union (EU), Canada, South Korea and the United States (US), and Gendered Innovations has expanded into Silicon Valley. A lot of interesting work is happening behind the scenes to integrate gender into the design process of all the products made in Silicon Valley. Currently, only two out of 26 of Gendered 
Innovations' case studies are devoted to the South (the developing world), but Gendered Innovations is very interested in expanding those perspectives, especially related to sustainable development, in the South. The operative question for Gendered Innovations is whether the creative power of sex and gender analysis can be harnessed for discovery, and whether considering gender adds a valuable dimension to research and takes research in a new direction.

Governments, universities and corporations have taken three strategic approaches to gender over the last 30 years. The first two involve fixing the numbers of women, which focuses on increasing the numbers of women participating in science and engineering, and fixing the institutions, which promotes gender equality in careers through structural change in research organisations. It is important that these two activities and policy directions should not be confused with fixing the knowledge, or gendered innovations, which stimulates excellence in S\&T by integrating sex and gender analysis into research. The third approach has to do with doing research wrong and thereby costing lives and money. Between 1997 and 2000, ten drugs were withdrawn from the US market because of life-threatening health effects. Eight of these showed greater severity for women. An analysis of the US Women's Health Initiative Hormone Therapy Trial found that for every $\$ 1$ spent on this research, $\$ 140$ were returned to taxpayers in healthcare savings, and the study also saved lives. More metrics are needed, mainly because policymakers like them. If a good example could be developed for the developing world about how integrating sex and gender into the research brought better research (and financial savings), it would be very influential.

Gendered Innovations' website (https://genderedinnovations.stanford. edu) is free and globally available. Methods for sex and gender analysis were developed working with the scientists and engineers in creating methods for science and engineering. Anyone can use these methods. The website also gives 26 case studies (concrete, practical illustrations) on how integrating sex and gender into research creates excellence, in the areas of science, health and medicine, engineering, and environment.

Examples of the case studies were presented:

- Machine translation

This is about computer science, natural language and processing. It was identified that Google Translate defaults to the masculine pronoun because 'he said' is more commonly found on the web than 'she said', and subsequently discovered that students in the engineering department of Stanford University are not taught anything about gender analytics. As a result, Stanford created a 
Faculty College of interdisciplinary professors to integrate enough knowledge of gender into the basic engineering courses so that the mistake will not be made by the engineers in industry. If other big companies started product development research by incorporating gender analysis, new innovative new technologies, software and system would be conceived. This unconscious gender (race, ethnic) bias from the past amplifies gender inequality in the future through the tool that is being used. Further work is being done in the area of algorithms and gender.

- Sanitary napkins (the study is currently being peer reviewed and is not yet on the website).

It was identified that women in rural India have no menstrual hygiene products and subsequently found that a company had invented a low-cost machine by which a group in the community in rural India could manufacture sanitary napkins and sell them at a low cost to other women in the community. This solution has two benefits: it provides women in rural India with menstrual hygiene products and provides jobs in the community. However, sanitary products worldwide are an environmental burden in terms of waste produced. Menstrual cups offer many benefits and are the solution to this problem. It is recommended that in India and other countries that do not have sanitary products, the menstrual cup, a much more sustainable solution, be adopted. A number of projects have studied introducing menstrual cups to school-aged girls in South Africa, Uganda, Kenya and rural India.

The website highlights areas of policy that work together: granting agencies, editors of peer-reviewed journals, educators and corporates.

- Granting agencies: These agencies have a huge amount of power and can ask applicants to explain how sex and gender analysis is relevant in their proposed research.

- Editors of peer-reviewed journals: Journals should not accept articles that are not excellent in terms of sex and gender analysis for publication.

- Educators: Sex and gender analysis needs to be integrated into university curriculum.

- Corporations: Gendered Innovations is running a series of techroundtables for leaders in Silicon Valley.

\section{QUESTIONS AND COMMENTS}

Mr Perkins: I am curious about the Faculty College of interdisciplinary professors created by Stanford University to integrate gender into the 
core curriculum. Was there resistance to this? If so, what tactics were used?

Response, Prof Schiebinger: In the US, it is new to try to integrate gender into engineering. We have been trying for a long time to integrate gender into medicine. The resistance is that the Nobel Prize Winners at Stanford and elsewhere say that more chemistry, and not gender analysis, is needed. I think it is criminal that this is not in medical school, especially as legal action is so common in the US. I am thinking of bringing a class action case of women against medical schools because they do not teach physicians how to treat women. The Faculty College (an interdisciplinary group) was funded by the administration for a year. We met to strategise how to get the information into the curriculum. It was necessary to work with every professor and show them (in their particular field) something they can easily integrate. Engineering professors are very unconscious about the gender aspect, but they are willing to listen. Our President and Provosts are very aware of the problem and willing to help. I am hopeful that we can bring about this change, but it takes a lot of effort for professors who were trained many years ago to learn something new. We have to find an easy way to help them do this.

Prof Abreu: How did you gather all the examples/case studies?

Response, Prof Schiebinger: We started with the examples that everybody knows about, only in science and engineering. We did heart disease in women and osteoporosis for men as a balance to heart disease. We did not do the basic research but summarised changes that had happened in the culture. We then worked with the European Commissions and several of the examples came from their grand societal challenges. My students brought questions and examples. We prefer working with funding agencies, grand challenges, big social problems and very technical things where there appears to be no gender. 


\section{PANEL 1}

\section{Gendered Research and Inclusive Innovation in the University Strategy (Prof Simone Buitendijk, Vice-Provost (Education), Imperial College, United Kingdom (UK))}

Universities and networks of universities are going to be pivotal in the GenderInSITE movement. The presentation looks at how institutions can become different places and change the world, and makes the case for gendered and inclusive research to be explicitly at the core of the university strategy.

There is a huge amount of bias in academia, particularly in terms of recruitment and retention of students and staff, teaching and curriculum, knowledge production, and societal impact. Social class has an impact on the opportunities for young people to access tertiary education or the best universities. Universities need to do a lot better to ensure that those from disadvantaged backgrounds are able to flourish and use their talents optimally. This can be done through thinking about the curriculum and teaching needs to be done differently, taking into account the realities of a diverse society and seeing students from different backgrounds as an asset rather than a problem. There needs to be much more awareness of diversity, inclusion and equality.

The way universities behave has a huge impact on how their staff feels. Women in male-dominated fields do not get promoted and/or have the same opportunities as their male counterparts. Women of colour are very under-represented in prestigious universities globally. This means something for the students that are being taught, for the research that is being done and for societal outreach.

Bias in knowledge production raises questions about what research is being done, who it is being done for, and how it can be used to better the world. It affects the core of what universities do, but in particular affects staff and students on an individual level, as well as the institutions in terms of culture, teaching, research and societal impact. On a global level, bias in knowledge production affects entire populations and the SDGs. University leaders need to think about different incentive structures to develop the innate drive of all academics to change the world, and enable them to do that at a much better level.

It is important to realise that there is something in today's academia that is firmly founded in a belief in meritocracy and recognising excellence, and help researchers to understand that universities are 
not as meritocratic as they think they are, and that it is very damaging to believe that the best people are in the highest status jobs. Institutions need to think beyond their own 'bubble', not about them versus 'the other', and encourage institutional empathy.

Individual bias relates to gender, ethnicity, social status, disability, sexual orientation and age, and the intersections of these. On an institutional level there is global bias with regard to the status of institutions and the rank of institutions in terms of world rankings, which are often related to geographical location and the field of research. Peer review is at the heart of academic competition and is biased. The global system around universities and what research they produce is hugely biased. This affects the focus of SDGs. The 'us' versus 'them' (the rich West versus poor South) approach cannot continue. All the global challenges affect everyone, although they impact countries differently, and have specific gendered impacts for example, in relation to climate change and the refugee crisis.

Inclusive research and innovation at universities will attract a broader group of talented students, change the discussions and culture in the classroom, provide a diverse student body, high-quality degrees and career options, attract and retain a broad group of researchers, stimulate institutional empathy and create a research environment with multidisciplinarity and global societal impact. Gendered/inclusive research and innovation must be promoted with urgency because global challenges cannot be resolved without inclusive knowledge production. In addition, there is no systematic consideration and mainstreaming of gendered and inclusive thinking in the research process, and researchers, university leaders, policymakers, politicians, research funders and (even) global organisations lack awareness.

Global change in knowledge production can be reached by putting gendered and inclusive research and innovation at the heart of the university strategy, challenging existing individual and institutional reward systems and incentivising inclusive research and teaching collaboration between universities globally.

\section{Applying a Gender Lens to Science, Technology and Innovation (Ms Dong Wu, Chief, Policy Review Section, Officer-in-Charge, Science and Technology Section, UN Conference on Trade and Development (UNCTAD))}

The CSTD is a subsidiary body of the UN Economic and Social Council (ECOSOC). It was established in 1992 and the UNCTAD is the Secretariat 
for this Commission. It has 43 member states, with South Africa as its most recent addition, and its main roles are:

- To advise the ECOSOC and the UN General Assembly on issues related to S\&T for development. It does this primarily through the analysis of priority themes that are selected on a yearly basis. Recent years' themes have been related to the specific SDGs and new innovation approaches to meeting the SDGs, such as missionoriented, pro-poor, grassroots innovation.

- To advance understanding of STI policy through the sharing of examples, best practices and lessons learnt. This is done primarily through peer review of national STI policies.

- To provide follow-up to the World Summit on the Information Society (WSIS). The Commission studies technological developments in the area of information communication technologies (ICTs) and their impacts on the economy, society and culture, particularly identifying opportunities and challenges for developing countries.

The CSTD meets once a year and the outcomes of its meetings become draft resolutions, which are essentially recommendations for action by national governments and international organisations. The CSTD is the only ECOSOC commission that has a Gender Advisory Board, which is co-chaired by Prof Shirley Malcom.

Gender has been very much in the DNA of the CSTD from its inception. In 1995, the Commission set up a Gender Working Group that produced a publication called Missing Links: Gender Equity in Science and Technology for Development. It made two important observations: that there are serious obstacles preventing girls and women from accessing science education and pursuing careers in S\&T, and that technical change was benefiting men more than women largely because S\&T policies and programmes did not explicitly recognise the genderspecific nature of development.

The Gender Working Group proposed seven Transformative Action Areas. This work complemented a wider effort to put gender and science on the policymaking agenda. In 1995, the Fourth World Conference on Women made special reference to S\&T in their Beijing Platform for Action. The seven action areas are intended to support governments in implementing policies and programmes that will contribute to gender equality. They are:

- Gender equity in S\&T education.

- Providing enabling measures for addressing gender inequalities in scientific and technological careers. 
- Making science responsive to the needs of society: the gender dimension.

- Making the S\&T decision-making process more gender aware.

- Relating better with local knowledge systems.

- Addressing ethical issues in S\&T: the gender dimension.

- Improving the collection of gender-disaggregated data for policymakers.

An eighth Transformative Action Area, promoting equal opportunities for entry and advancement into larger scale science, technology, engineering, and mathematics (STEM) and Innovation Systems, was added in 2006.

In 2011, at a time when there was a renewed interest in issues of sustainability and inequality at a global level, UNCTAD and the CSTD Gender Advisory Board revisited this topic. Building on the Missing Link publication, they produced a second publication on this topic entitled Applying a Gender Lens to STI. The report brings together years of research into the different dimensions of gender in STI and connects the extensive evidence of gender-based patterns and inequalities and their implications in STI. In doing so, it makes the case for improving gender sensitivity throughout STI policymaking processes. It proposes using a gender lens, which spans three dimensions, namely:

- Science for women, which involves developing S\&T that support women's development and livelihood activities, especially in areas such as agriculture, water, energy and transport; reduces women's workload and increases the value of the productive activities by improving quality and efficiency, thereby increasing incomegenerating opportunities and improving health and quality of life for women.

- Women in science, promoting gender equality in STEM education, careers and leadership.

- Women in innovation, encouraging and supporting the role of women in innovation systems at the grassroots and national levels, removing constraints on the productive capacity of women, and recognising them as both users and creators of knowledge and technology, and as valuable economic actors. Strategies in this area aim to ensure women have equal access to education and training, capital and markets, encouraging and supporting the role of women in innovation, enterprise and entrepreneurship.

These dimensions are highly relevant today. Earlier this year, when the Commission on the Status of Women was addressing the issue of the 
world of work, it was learnt that women take home one-tenth of the global income, but perform two-thirds of global working hours. They do twice as much unpaid work as men (such as collecting fuels, cooking, caring for the old and young, doing subsistence farming). There is an urgent need for technologies and innovation that support women's livelihood activities, especially improvements in the distribution of affordable household appliances to relieve women from the household burdens.

The advent of ICTs has provided important opportunities. For example, women entrepreneurs in developed and developing countries have new opportunities to strengthen their businesses and become more effective. ICTs provide women entrepreneurs with ways to reach customers and grow their businesses in ways they could not do before. While there is enormous potential, there are also significant impediments to women's participation in the digital economy. The worsening digital gender divide is a worrying trend, with 2016 data showing that women worldwide are $12 \%$ less likely to be online than men, with the difference reaching $31 \%$ in least developed countries (LDCs).

One of the main activities of UNCTAD that supports of the CSTD is STI Policy (STIP) reviews. To date, 16 reviews have been conducted. The main objectives of the STIP reviews are:

- to provide diagnostics of the effectiveness of STIP framework and to provide policy advice to countries;

- to identify the gaps, strengths, weaknesses and challenges in national innovation systems with a special focus on the policies, incentive measures, institutions, infrastructure and regulatory environment necessary to encourage local technological learning and innovation;

- to identify practical actions that foster technology upgrading and stronger innovation capacity at the corporate, industry and national levels.

Secondary objectives of the STIP are to support effective integration of STI policy in development strategy; to promote awareness of role of STI policy in sustainable development; and to stimulate multistakeholder discussion in the country on STI policy for sustainable development.

The review process involves identification of the key entities involved in STI, data collections (qualitative and quantitative) and diagnosis. Discussions are held with local counterparts to clarify any issues and advanced drafts are discussed with stakeholders. The reviews are peerreviewed by the CSTD and contain policy recommendations that are 
proposed in consultation with local partners. A follow-up is done after a few years to assess the extent to which the recommendations have been taken up.

Recent reviews aimed to explicitly reflect gender dimensions through gathering data, particularly data related to education and vocational technical training and in terms of policies that target women. It was observed in the early stages of this work that fundamental issues need to be addressed within the STIP review framework as it fails to capture information from the informal sector and 'below the radar innovations' due to the lack of data, and requires a systemic approach.

A series of workshops (including one in Pretoria on 20 and 21 September 2017) organised by the Transformative Innovation Policy Consortium (TIPC) aims to produce an Innovation Policy 3.0 document that will incorporate the issues of sustainability and inclusiveness.

\section{Impediments to the Full Participation of Women in Innovation (Prof Jennifer Thomson, GenderInSITE Co-Chair, President, OWSD; University of Cape Town, South Africa)}

Innovation is needed for poverty alleviation. STI provides the tools for sustainable development but it is not happening in developing countries and needs to increase. Women bear the brunt of the effects of poverty and it therefore makes sense not to exclude $50 \%$ of the population (women) in finding solutions. Women approach STI differently. They (and some men) tend to be multidisciplinary and non-linear, and will produce the best innovations. Men tend to be single-minded and this is good for ratings and funding. In terms of managing STI, women have a gentle touch and are natural bridge-builders. Innovation requires working together and poverty reduction requires innovation.

A gender lens on research and policy development towards innovation is essential. It has been shown that rats in laboratories respond with fear when male technicians enter the room, but do not respond with fear when a female technician enters the room. In agriculture, women's crops feed people, while men's crops make money. Who decides what gets researched? Who makes decisions on water infrastructure development and water reticulation, and who fetches water? In terms of energy development, who will do a study about how to bring the best energy to cities or to rural areas?

With the migration to cities, it is the elderly and children who remain in rural areas. In terms of transport improvements, is the bigger priority 
mass urban transport or improving access to markets by rural farmers? Over-population would be addressed through educating the women.

Much can be done to remedy the situation. Academies can assess the situation, look at sex-disaggregated data on women in STI and report on it. They can also address the gender lens through the fellows elected to the Academy, and through its committees and projects. Every STI government department should have a Gender Desk. Rwanda has a Gender Monitoring Office that targets and monitors what is being done.

A few years ago, the South African Minister of S\&T, Minister Naledi Pandor, put out a call for Research Chairs as part of the South Africa Research Chair Initiative (SARChl) that was only for women. When announcing the 42 new Chairs, the Minister addressed the concern that they had not been initiated by their respective institutions, but by the DST. OWSD has been involved in ensuring that these Chairs are enabled to reach their fullest potential.

Some of the practical ways to combat gender inequality in the STI research environment include:

- Holding important meetings during school hours.

- Extending cut-off for 'young scientists' from 35 (suitable for men) to 45 for women.

- Requiring gender representation at conferences, workshops, committees, etc.

- Providing laboratory support during maternity leave.

- Paying for child-care costs for women with pre-school children.

- Allowing women who are funded to attend conferences to use funds to take children and carers.

- Providing a family room at conferences.

- Establishing gender equity committees at all research institutions and subjecting them to monitoring and evaluation (M\&E).

It was noted that the DST's new White Paper of S\&T only provides platitudes when making reference to gender.

In conclusion, innovation is required for development to alleviate poverty. Women need to be included in priorities because they know what is needed. Those involved in STI should capitalise on the fact that women approach science and its management differently. There is a role for academies in revealing and using the gender lens 
to solve relevant problems. It is essential to have Gender Desks in all STI government departments, and there are numerous practical, easily implementable ways to improve women's participation in STI.

\section{Creating Improvements in Innovation for Women (Dr Sharifah Maimunah Syed Zin, Director, International Science, Technology and Innovation Centre for South South Co-operation (ISTIC))}

An analysis of all the SDGs shows that women play a pivotal role in achieving almost all the goals. The fact that one of the goals (SDG 5) focuses solely on gender equality shows that a lot more still needs to be done. There have been improvements in education. The enrolment of girls at primary, secondary and tertiary institutions has increased, but women tend to not occupy high-level decision-making positions. Global figures from the UN show that only $24 \%$ of senior management positions are occupied by women. Despite all the various actions being taken, there are still many challenges and there have been many calls for the need to generate innovative ideas and creative solutions to achieve gender equality.

Innovation has to do with new products or processes, or old products and processes put together in new ways. If innovation is about change, it must benefit men and women equally. Literature shows that innovation can change lives and improve the well-being of women in terms of health and nutrition, as well as the life span of women. Entrepreneurship has improved self-confidence among women and innovation has enabled women entrepreneurs to become more influential, venture into unchartered territory, enter markets rapidly, be more mobile and resolve problems. However, men and women are socially conditioned to see things differently. Problems and perceptions are gendered and gender equity can be judged differently by men and women. Equality means equal amounts of something and equity has to do with fair distribution and is not necessarily the same as 'equal'. Involving women will contribute to resolving problems relating to these issues. Some examples of innovators' lack of consideration of the gender dimension and of instances where the gender dimension was applied are:

- An 'online post office' was introduced in Malaysia some years ago to help the disbursement of pension to villagers, widows in particular, so that they do not have to spend money and time taking a bus to the nearest town. However, the women's preference was to go to town to collect their pension and they viewed this as a regular outing and an opportunity to visit friends, go shopping and attend to other business. 
- A solar energy project involved uneducated grandmothers who were sent to India to do a course to learn how to fix solar panels that were used in the villages, using colour coding. Women were chosen for the project because they stayed in the village while most of the men worked in the towns. The project enabled numerous households to have electricity and is an example of successful application of the gender lens.

- Many of the school buildings in Malaysia are more than two storeys high. The plight of pregnant and elderly teachers having to climb staircases is not taken into consideration.

- Women are often victims of smash and grab crimes, where handbags are stolen out of their cars. Although there are numerous innovations in the automobile industry, not one has provided a safe place for women to put their handbags. If women were involved in the design of automobiles they would think about such an innovation.

How do we ensure that the gender lens is incorporated when developing innovations? Can men and women learn from each other? Are lenses absolute? Can we change the way we see things? It is suggested that in order to improve innovations for all women, they should engage in STI. Women are as important as men in contributing to development and they play central roles in sectors that affect well-being. Women are more prone to health risk, pollution and physical injury. STI is important in addressing challenges faced by women in fulfilling the roles of food producers, care givers and social educators, and women can better understand STI-related issues that affect women. Educating women creates a science literate society and contributes to industry.

However, women often hesitate to speak out because of a lack of confidence and education. Capacity building to provide women with communication, management, leadership, entrepreneurial and ICT skills can help them overcome this problem. Opportunities could be created for career development by providing courses at tertiary level and at the workplace, through establishing networks between women scientists and sharing success stories. There should be dedicated incubators for young women in STI, and schoolchildren should be prepared for the $4^{\text {th }}$ Industrial Revolution and the future job market by offering equal opportunities to girls and boys. Allowance must be made for more women leaders in government, industry and decisionmaking bodies. Women in leadership positions are more able to voice the needs of women in general. 
ISTIC has capacity building programmes for women in STI (one of the centre's priorityareas) with afocusonmiddle-levelmanagement, offering leadership management and entrepreneurship/techpreneurship that provide skills to enable researchers to commercialise their products. It is essential to provide women in middle-level leadership with skills they require in order to advance their careers. It is also necessary for women to be given the appropriate knowledge and skills so that they can access positions of influence and decision-making, enabling them to focus on gender issues, particularly in the workplace and at middlemanagement level.

\section{QUESTIONS AND COMMENTS}

Prof Schiebinger: Where can Ms Wu's work on sustainable cities be found? What are some of the highlights of that work?

Response, Ms Wu: Usually, after the CSTD has examined priority themes, we produce a research paper that gets published as part of the current series on STI. There was a discussion on gender particularly with regard to the use of geographic information systems to map the genderrelated issues in peri-urban communities (slums). The gender-related issues in these communities have to do with energy, water, growing vegetables and more. The citation for the work will be distributed to participants of this workshop.

Prof Malcom: I know that there has been work in terms of genomic sequencing of a lot of crops. It is not clear that the crops that women grow are being targeted for sequencing?

Response, Prof Thomson: This is correct.

Prof Abreu: I would like to ask Prof Buitendijk about the League of European Research Universities (LERU) network of universities. How global is its reach and would be interesting to try to connect more regions?

Response, Prof Buitendijk: LERU is creating a network of networks. They are starting to collaborate with the Chinese, the Australians, as well as the African Association of Universities, connecting all the networks of top research universities. This kind of new platform could be very useful if we are trying to connect globally in more focused research and impact when talking about the SDGs. LERU is modelling a network of networks on a network of research funders. If the new global network of research universities could be linked to research funders and many 
other networks, targeting the meta-level to not only inspire individual researchers, but also inspire individual universities and then networks of universities and other collaborators, it would be a great way to get ahead quickly. The first time that the network of networks came together was in China and the second time was in The Netherlands when the theme was involvement of Social Science and the Humanities in Science Production. Gendered Innovation could be the theme of the third meeting when they get together sometime in the future.

Prof Diab: A network of African research universities called the African Research Universities Alliance (ARUA) has been created recently.

Prof Abreu: There is also a Latin American network of research universities.

Dr Comins: What have you learnt about the processes of taking that research into the implementation phase? What are the mechanisms and who is involved in those particular processes, many of which require both hard and soft sciences working closely together?

Response, Dr Maimunah Syed Zin: We have a programme on how to further develop the research studies and commercialise them. The researchers are trained as entrepreneurs/technopreneurs. We publish the publication together with TWAS. This is not only for women and we are trying to come up with a publication on women inventors.

Response, Prof Buitendijk: This is the most crucial question for this workshop. Of all the successful innovations, most are due to the implementation and not because of the technical elements. This collaboration and the notion of how the product will be used needs to be built into the research phase, instead of waiting until there is an invention and then start thinking about what to do with it. Doing this globally should become more the norm.

Response, Prof Thomson: Involve the social scientists as this will enhance acceptance of the innovation. The mechanisms for bringing to scale are critical in the implementation phase. This is what institutions such as TIA are doing.

Response, Ms Wu: A lot of the new technologies are being developed at breakneck speed, but the inventors are not talking to social scientists. It is crucial to think of ethical issues and the impact on employment, for example. A multidisciplinary approach is extremely important. The CSTD's STIP research is gender-lensed. When we go on 
fact finding missions we often find that there are no women involved in the development of new technologies. There is a huge gap between bright ideas and recommendations, and reality. We should look at how to integrate the gender lens into reality.

Response, Prof Schiebinger: The idea of social scientists working together with natural scientists and engineers is very important. Companies like Google and Facebook realise now that they need social scientists and humanists. This is new in the last few years. Social scientists also need to meet the natural scientists and engineers halfway. The most important thing is that we understand each other's language. The Gendered Innovations website makes a point of avoiding jargon. Feminists also have to be careful that we always speak a language that communicates across broad groups. We need to bring knowledge back to a time when there was no division between social scientists and natural scientists.

Prof Malcom: We have been talking about settings where there is public support for gendered innovation. We have not talked as much about corporate policies insofar as how they mentor for leadership and move people up within the entity, how they look at their boards and make decisions about what they develop and do research on, and we need to look at the private sector.

\section{PANEL 2}

\section{Innovation Beyond Stirring: Reflecting Dimensions of Gender in What Works (Mr Nick Perkins, Lead Technical Advisor: Development Communication and Extension, Centre for Agriculture and Biosciences International ( $\mathrm{CABI})$ )}

The title of this presentation is a reference to an article entitled, Quotas: Add Women and Stir? that was published by an Egyptian researcher in 2010. Her contention was the narrative that if the numbers can be fixed it can be transformative is not necessarily true. She was concerned that the kind of legislative bills that supported quotas for women in public participation were a convenient conflation of allegiances and did not necessarily reflect a very progressive idea about women's participation and parity. She wanted to explore this in her article by looking at empirical evidence around this issue. It is necessary to essentialise the genders, women and men, because it has been seen that this is not always the case. Women themselves will have different political agendas and make different choices about how they see 
transformation and what is important for transformation. Another important issue behind this article is that examining quotas moves away from women in development, the idea that if women are targeted it is good for efficiency, moving towards gender and development (the relationship and the way that people talk about the sexes, the roles that men and women have and the social norms), which reproduces the issues that are being addressed. It is not per se about men or about women but about the social norms.

In terms of empirical research around innovation, a very interesting study was done in $2013 / 14$ by a Xiaolan Fu, a professor of technology and international development at the University of Oxford. She did a survey in Ghana of around 500 firms. About half the firms were in the informal sector. She found that there was quite a lot of innovation taking place in Ghana and most of the innovation was in the informal sector. Also that the innovation was largely incremental and the diffusion was nationally focused coming through national networks of local entrepreneurs within the country. The innovators had very little formal contact with public bodies and with academies, universities and more formal research organisations. She was talking about products, services, marketing strategies and management inputs.

Another article from SciDev.Net, about a study that will soon be launched in Ghana, makes a point that although the number of female-headed and female-funded small firms typically classified as technopreneurships can be monitored, very little research has been done on this issue. Caroline Moser, an anthropologist who has done a lot of work on gender and development, says that there can be awareness about gender, but assumptions can be made that reproduce the inequalities and inequities that are being challenged. An aspect of major importance will be missed if there is a lack of mindfulness and care to monitor where this innovation is happening.

The implications for growing a culture of innovation that reflects an awareness of gender, and particularly the broader ambitions around gender and development, is a project that has a fundamentally social and political agenda. When thinking about the informal sector, the factor that comes to mind is that women tend to gravitate towards opportunities for self-employment for various reasons, but there are also things that are familiar from the perspective of gender planning over the last few decades where the notion is that a lot of problems are due to culture (a political agenda). This has resonance with innovation in the context of the informal sector, which is a particularly fertile space for innovation and high in participation levels for women, but falls outside 
of the purview of most public institutions let alone corporate institutions when planning innovation. This is where there has been a move towards grassroots programming in the context of gender programmes. Naila Kabeer's book, Reversed Realities, talks about grassroots and gender programming and is recommended reading.

Some key lessons from the last few decades of grassroots programming are identified and suggestions made about the way gender innovation is thought about in those spaces where gender innovation is particularly fertile, with respect to:

- Participatory modes of needs identification.

- Interventions need to compensate for the exclusionary nature of most institutions.

- Consider how women are framed, as needy clients or constrained actors.

- Focus accountability 'downwards' and not around globally preconceived agendas.

- Policy and political leadership matter.

\section{Gender and Innovation: An Asian Perspective (Prof Miyoko Watanabe, Deputy Executive Director, Director-General of Centre for Science Communication and Director of Office for Diversity and Inclusion, Japan Science and Technology Agency)}

The Asian perspective is epitomised in the most popular Japanese literature, The Tales of the Heike, which says, "The sound of bells echoes through the monastery at Gion Shoja, telling all who hear it that nothing is permanent. The colour of Sala trees gives witness to the truth that all who flourish must necessarily perish". Most Japanese and Asian people are one with nature (the truth) reflected in the four seasons, and are polytheists.

Gender Summit 10 (GS10) Asia Pacific took place in May 2017 in Tokyo under the theme, Better Science and Innovation through Gender, Diversity and Inclusive Engagement, with the participation of 603 people from 23 countries. The summit focused on the Asian perspective. The event was funded by about 70 sponsors and 114 organisations contributed to the organisation of the summit, which aimed to redefine gender equality. The keynote speakers were:

- Prof Juichi Yamagiwa, President, Kyoto University, Japan, who spoke about the origin and biological base of human society. He explained the long history of human society and said that the characteristics of human society are not based on biological kin relations, but on 
cognitive relations through caretaking after birth. He concluded that future perspectives of gender and diversity should reconsider the greater flexibility and resilience in family formation.

- Prof Angela Ki Che Leung, Director and Chair Professor, University of Hong Kong, who enquired whether Confucianism is to be blamed for gender inequality in East Asia. Although the common belief is that Confucianism, as a patriarchal system, confined women within homes and hindered social activity, she found that Confucianism gave women the important role of managing their families, and contributing to society's economy, politics and so on through families. A 'husband pocket money' system is practised in Japan. Men give their entire salaries to their wives to manage and are allocated fixed amounts of money. Confucianism became a target of attack in the late $19^{\text {th }}$ century when it was challenged by Western industrialisation and imperialism, and as a result, a lot of Asian customs and perspectives were replaced with modern Western values (such as science and democracy).

- Dr Chieko Asakawa, IBM Fellow, IBM Japan, who spoke about innovation created by the dreams and needs of a blind researcher. She developed a Netscape browser plug-in and the IBM Home Page Reader, the most widely used web-to-speech system available, and contributed to innovation not only for blind people but for everyone. She was also developing new technology that would help blind people to walk unaided.

- Dr Reiko Abe, President, Oriental Consultants India, who spoke about her experiences as a female civil engineer in charge of metro construction in India. Although she experienced many legal restraints in relation to her career in civil engineering in Japan, she has been very successful in Asia, practising as a metro construction managing director in India where she introduced safety standards for construction workers.

The summit involved several working groups and 21 people from eight countries contributed to making the recommendations, which formed part of the summary of the event with the title, Better Research and Innovation through Diversity and Gender Equality (BRIDGE). The recommendations were:

- Gender equality is an essential determinant of societal sustainability and well-being and affects how STI can improve people's lives.

- Gender equality should be integrated into the implementation of all 17 SDGs, because gender equality within STI provides a BRIDGE through which targets of all 17 SDGs can be connected to enhance implementation success of the UN SDG agenda. 
- Gender equality in the context of SDG targets must recognise the influence of human and societal diversities, and, in particular, how science and societies define and BRIDGE the roles and attitudes to women, girls, men, boys, ethnicity, race, cultures, etc. Gender Equality 2.0 should be adopted by all stakeholders, including industry when planning and implementing actions to achieve sustainability.

\section{Gender, STI and the SDGs - South African Developments (Mr Imraan Patel, Deputy Director-General, Socio-Economic Innovation Partnerships, DST)}

The issue of the intersection between gender, innovation and development is growing in importance. It is necessary to acknowledge the gap between innovation and development. The SDGs, although not perfect from a gender perspective, provide a firm foundation and a step ahead of previous global efforts to interface between gender, development and STI. There is a much more focused attempt from the STI community to look at the role of STI in achieving the SDGs. One SDG deals specifically with gender equality, but is also comes through in other SDGs. More needs to be done to unpack what it means, particularly in areas such as industrialisation. The elements of gender are strongly present in the SDGs on education, health, etc. In South Africa, gender intersects with race, location, class and other factors. The experiences of a black woman in a rural community are very different from that of a white woman in an urban setting. This has to be taken into account in the way in which responses are formulated.

"Science knowledge, research methods and practices and the innovation system are not free from gender bias. Reversing these biases requires global and national action." This comes from the South Africa's NDP. There is the debate about how to institutionalise gender. The gender mainstreaming approach is proving too difficult to put into operation because it demands a high level of gender expertise and political commitment. In the absence of these, alternatives need to be considered.

The DST will work with ASSAf about how to institutionalise the Seoul Gender Summit Declaration (an outcome of the Gender Summit 6 in Korea in 2015) in the South African context, as it sets out a good basis for action.

Some of the building blocks in terms of gender in South Africa are:

- The growing appreciation of gender in the socio-economic cluster of government. Through the work of the Department of Home 
Affairs there is a requirement for all data to be disaggregated by gender. This has to be continuously reinforced. There is also a requirement in terms of the targets set by government departments to be explicit about the impact on women.

- The Gender Desk in the DST, and steps have been taken towards developing Gender Desks for the entire NSI.

- The experiments with gender budgeting. There are important methodologies around resource flows and it is therefore crucial to get a handle on the national budget from a gender perspective, the resources flows will shift. For example, there is a need to look at large-scale incentive programmes and ask the question of how they impact on women.

- The DST's inclusive development approach, which provides the basis to support all excluded communities.

- The an attempt to consider transformative innovation policy approach in terms of framing the DST's new White Paper on S\&T, to focus on underlying social technical systems. This approach provides a good basis from which to adopt the gender lens.

- Important changes that were beginning to be seen in the demographics of the research and innovation communities.

- Efforts that were being made to improve gender parity within the DST's flagship projects, such as SARChl and the Centres of Excellence.

- Tentative efforts that have been made to deal with institutional blockages that disadvantage women researchers.

- The changes that have been made to the physical spaces for research.

- Strong international co-operation in a wide range of areas, including gender in STI, and as an anticipated outcome of this workshop.

- A number of targeted efforts at girl learners, although they are not necessarily at scale.

Some of the areas of challenge are:

- Early childhood development and the schooling system: A lot of work needs to be done in this space. The schooling system is not designed to accommodate the different ways that girls and boys learn.

- Curriculum reform: There is no fundamental gender-based research that can be used an innovation system. A recent move requires all policies to go through a socio-economic impact 
assessment. The gender divide aspect of policies and the assessments of policies need to be strengthened.

- Role models: There is a lack of role models in science and engineering, in particular role models that rural girls relate to in terms of STI.

- The working environment and culture.

- Responsible research in innovation: This has to do with the process of people applying for grants for research funding and the need for funding agencies to begin to change mindsets.

- Transdisciplinary approaches: It is necessary to move beyond multidisciplinary approaches involving social scientists and natural scientists to transdisciplinary approaches to include users in the research process.

- The low level of deployment of available innovations, particularly those that reduce drudgery in rural agriculture, for example.

Issues that require attention in the global perspective include:

- The financing of innovation, including business models, and its impact on the upscaling and commercialisation of innovations.

- Transitions and sequencing.

- The tyranny of current metrics, such as gross domestic product (GDP), which requires rethinking.

- The future challenge of young men including the increasing marginalisation of young men, social norms and constructs affecting boys and men.

\section{QUESTIONS AND COMMENTS}

Prof Abreu: What does it mean to have interventions for the majority? This relates to the point about GDP that does not always capture the informal sector, and the recognition that we tend to value what we can measure rather than measure what we value. It seems that we put women into a box they cannot get out of just because of the way we measure.

Response, Mr Patel: There have been a lot of debates around GDP, for example, but they have not had traction. It is through forums like this that these challenges can be confronted. The impacts are clear and for all to see. Alternatives are needed in terms of the metrics and the way we measure. There needs to be a global effort run by young people to re-imagine the metric, particularly the growth dynamic, which is not the best measure for sustainability. A central drive is needed to 
confront key issues such as this as part of the gender-based agenda for sustainable development.

Response, Mr Perkins: I have a very political perspective on the GDP issue. No-one took it seriously that there could be something else, until the financial crisis of 2008/09 when there was an admission that something else should be thought about. In terms of the issue about intervention, there is a notion that intervention should be for as many people as possible, particularly in the context of global development (this idea of the silver bullet must be challenged), and it is necessary to think about context and less about scale. We see lots of examples of inventions for the many in social and digital media.

Response, Prof Watanabe: GDP is a very important metric but not the only way to measure society. We should measure the other metrics, for example the process and the situation. GDP is just the result of processes. Both processes and the results should be measured. The same applies to gender equality. The gender ratio is a very easy way to compare numbers, but other metrics should also be used to measure gender equality.

Prof Abreu: The fourth participant is this panel, Guillermo Anlló, could unfortunately not join this workshop. He was going to present STEM and Gender Advancement (SAGA), a new initiative that should be looked at. It brings a very complete and holistic approach of how to have indicators on gender and STI. My comments to the presenters are as follows:

- To Mr Perkins: In my previous academic life, I worked a lot on the informal sector, particularly the garment industry. The informal sector is not a bubble and is sometimes very much linked to the formal sector. The whole system should also be looked at.

- To Prof Watanabe: She brought in the issue of culture. This really needs to be looked at in the way we approach gender, innovation and science. This makes sense in terms of what GenderlnSITE is trying to do by having regional focal points that look through the local perspective to understand how it functions and alter the way that gender and innovation is perceived.

- To Mr Patel: I was part of the National Research Council of Brazil for a few years and I recognise all the challenges he mentioned. One very important issue was the need for disaggregated data on all the systems. This will allow us to see the problems and understand what is going on. Brazil has had an enormous capacity building effort over the last 60 years, with no gender policy. It provides around 2000 scholarships a year at all levels. Women are the majority of 
fellows in all the scholarships except one, the very prestigious senior research scholarship. Why is it that in some type of scholarships there are many women and in this one there are no women? We need to understand the processes and the way the scholarships are granted. Metrics allow us to see the problems, but also to ask questions about what is happening in the methodology.

Response, Mr Perkins: I take your point about the relation between the informal and the more formal sectors. I was signalling a cautionary note about where this innovation is taking place and that the informal sector is not strongly related to national networks or research sites.

Response, Prof Watanabe: We cannot control the culture trajectory, but if we neglect culture we cannot change society.

Prof Buitendijk: In terms of the metrics on economics and GDP, we should also look at the metrics for research and academic performance. There is a huge skewedness towards STEM, medicine and innovative science that require expensive laboratories and many resources, while the social sciences and humanities often do not get measured well. University rankings look at a type of research that is not multidisciplinary and does not include women. If we want change we need to change the ranking of universities' scientific prestige and reward system.

Dr Comins: I am trying to understand the contrast between the 'bottom-up innovation' which is often local, community-based and disconnected, and a national policy. Is the role of national policy a facilitation basis or a driver basis? If it is too much of a driver then it does not facilitate bottom-up. If it is too much bottom-up then some future thinking is lost. This could be a challenge.

Response, Mr Perkins: In gender planning there are people who are specialists in planning or specialists in gender. There are very few people in the science of scaling and particularly, expertise needed to go from the research in the laboratory or in the street to uptake. There is already a scarcity of capacity and skills in this regard. Combine this with the issue of understanding gender from the point of the broader humanity perspective. A lot of gender planning stumbles when it comes to culture. The issue then becomes how to reconcile the technical requirements with the political and social impediments. This is where a number of alternatives were developed within gender programming. One of them is the idea of taking on grassroots programming. In the Ghana study there was not national policy in place. A driver is not needed, but a facilitator is needed to support women innovators and 
provide them with economic emancipation and the ability to transition the innovation into something that has social impact. This has big institutional implications.

Response, Mr Patel: It is a bit of both. It depends on the context. I did not mention what the DST is doing in terms of grassroots innovation. We need to find out what is driving people. Strong direction is needed at times. The example was given about the call for women holders of SARChl chairs. The best approach needs to be determined at any point in time. Increasingly, it makes sense for a national government department to be playing an enabling role instead of giving strong direction.

Mrs Marais: With regard to transformative actions, South Africa has a strong policy environment, strong programmes and international representivity at women's events, we have key performance indicators that count women, but everything is still quantitative. There are not many measures that look at qualitative matters on the ground. All of our women activation is sexualised and about violence and lack of economic access and access to basic services. Entrepreneurs who are applying for funding for innovation in South Africa today, in particular the youth, are involved in activities that all relate to indigenous knowledge, cosmetics and health, and most of the applications come from men. These are viewed as soft solutions. We are rewarding patents and scientists, but there is not a lot of effort in respect of enablers and managers to actually drive programmes. We have achieved a lot, but are still at the policy level and have not moved to the action level.

Response, Mr Perkins: That is an interesting observation. It reminds me of the work done around strategic needs versus practical needs in gender. This sounds like a simple, helpful dichotomy while recent writing suggests that there is a very complex relationship between these two things. What you are describing is in the context of where South Africa is at the moment, thinking a bit about how you have a framework that allows these two things to speak to each other. 


\section{PANEL 3}

Gender and Research, New Results and Activities in Germany (Prof Martina Schraudner, Head, Fraunhofer Centre for Responsible Research and Innovation (CeRRI) at the Institute for Industrial Engineering (IAO), and Chair for Gender and Diversity in Technology and Product Development at the Technical University, Berlin (Via Skype))

The Fraunhofer Society is not a university but a research organisation that does applied research. It is funded by the government, from government projects and from direct project research with industry. This is the reason why Fraunhofer works in the spheres of engineering, life sciences and micro-electronics. Around 23000 people work at Fraunhofer and the various institutes are spread throughout Germany.

The topic of the integration of the gender dimension is important for Fraunhofer because it has a very small number of women researchers (about 15\%). Prof Schraudner does research about the promotion of women in companies as well as in research. There has been a shift in the reasons why companies and research organisations are interested in having women on board. A few years ago, gender equality and equity was about fairness and justice, and equal opportunities for women, which was written into law (where the focus is on support for the single woman).

More recently, the arguments for gender equity are based on the need for consumer awareness, organisational development and the attraction and retention of talents. Companies are interested in promoting women within their organisations. The arguments are much more driven by economics and changes in organisational behaviour in order to increase the number of women.

Top-down initiatives have the most impact to promote more women in academia. These initiatives and programmes are funded by the German Research Ministry. The Pact for Research and Innovation has to do with additional money given to the research organisations for having activities to promote women in science. One of the evaluation criteria for the Excellence Initiative is the consideration of gender equality policies. The Programme for Women Professors is a specific programme funded by the German Research Ministry to bring more women professors to the universities and research organisations with the aim of increasing the number of female professors and creating role models. The German Research Funding Organisation (DFG), which 
funds research at universities, has prescribed the Research-Oriented Standards in Gender Equality since 2008. This programme has a very strong impact especially on universities.

Shifts have been seen in relation to European, British and American funding of research projects since 2000. The DFG funds basic research at universities. Additional programmes for applied research are funded by the European Commission or the German and British Research Ministries that want to see societal impact of their research. This started with the European Programme 5, before 2000, and is an ongoing process that is becoming more and more important. Research that addresses societal needs is funded via these programmes and is referred to as 'broadening impact' in the US and the 'third mission' in Germany and 'responsible research and innovation' in the European programmes. This kind of more applied research can be addressed via paper work or socio-technical systems. Engineers have only recently begun to think about scientific-technical inventions as needing to address societal requirements, according to the specific needs and preferences of society in general, as well as of men and women.

Bringing the ideals of more women in research, more gender analysis in knowledge production and more highly trained women together with markets for science knowledge will help overcome the 'valley of death' and result in an improvement of the innovation ecosystems. Although doing science for science's sake is very important, there is also another very important part of research that involves doing research to benefit society, which could be done directly, through offering advice to government and to industry.

In conclusion, more women are needed in research in decision-making bodies, and a gender lens must be used to describe and evaluate the impact of research. More women as entrepreneurs helps address the gender balance in the innovation system as it changes mindsets, helps develop a shared vision with society and shapes the future collaboratively.

\section{Gender and Innovation at São Paulo Research Foundation (FAPESP) (Prof Ana Maria Fonseca de Almeida, Assistant Coordinator of Social and Human Sciences, FAPESP)}

FAPESP funding forms $1 \%$ of the total fiscal revenue of the state and provides stability and autonomy. All areas of science are treated equally, but the focus is on innovation and collaboration with industry. The result of this long-term investment in research is that $25 \%$ of the 
country's scientists (who are located in the state of São Paulo) create $44 \%$ of the scientific articles and supervise $40 \%$ of the doctoral theses concluded each year.

The S\&T system in the state of São Paulo has 74000 researchers working in higher learning, companies and in-state, federal and private research institutes. Expenditure on research and development in the state is $\$ 14.8$ billion (purchasing power parity (PPP)), by far the highest in Brazil, and among the highest internationally. São Paulo generates more articles than any country in Latin America.

Three main characteristics of the scientific environment in Brazil are:

- It is quite a new system. Research as it is known today was very rare in Brazil during the 1960s when graduate programmes were regulated by the state and became a focus of sustained investment, even during the political and economic crisis that followed.

- Most of the science institutional structures, graduate programmes, research funding and careers in research were created during the military regime in Brazil. At the time those investments were being made, the universities were under attack from some sections of the same regime. University professors were being persecuted and many of them lost their jobs and were forced to flee the country.

- As a result of this investment, research today happens mainly in government institutions, many of which are universities where professors and researchers are recruited as public servants, ensuring employment stability, career progression, competitive salaries and advantageous retirement benefits. The political dispute that took place in the 1960s through to the 1980s resulted in the political volatility of public universities today. Part of the faculty is suspicious of any kind of top-down instruction coming from government agencies and is aggressively involved in advocating the reduction of educational inequalities, mostly framed in terms of inequalities in social backgrounds and race. These issues have overshadowed the public discussion on gender inequality in education and in science.

FAPESP was established in the early 1960s and throughout the years it has developed a bottom-up approach to research funding in which the researchers themselves decide on the themes they pursue and the problems they tackle. One of the main contributions of FAPESP has been to provide a roadmap to better identify good research relying exclusively on peer evaluation based on clear and transparent practice. FAPESP has taken some steps towards addressing gender equality. For example, it has instituted six months maternity leave for 34 students who have received scholarships from FAPESP and is making a 
concerted effort to include women in all fields and all committees. The approaches adopted by FAPESP are particularly beneficial to women in that they:

- Contribute to building the autonomy of the scientific field by preserving the capacity of scientists to regulate themselves thus creating a barrier against the interference of politics in the scientific agenda. This is something that FAPESP cherishes, particularly in the context of Brazil's young democracy.

- Help level the playing field for those areas that have developed at a slower pace than others.

Gender as an analytical category has found some success in the Brazilian academic landscape. More than 1000 registered research groups have gender as an exclusive subject of study, but a lot still needs to be done about the incorporation of gender in science by Brazilian researchers. In terms of the participation of women in science in Brazil, the number of PhDs awarded to women has increased substantially to reach $54.4 \%$ in 2014 . Women fill $45 \%$ of the positions in public higher learning institutions, earning competitive salaries, and are likely to pursue careers in science. Elsevier's General Global Research Landscape (GGRL) puts Brazil ninth out of 12 countries where women comprise more than $40 \%$ of publishing researchers.

In terms of scholarly output and impact, women publish an almost equal number of papers as men; $48 \%$ of the papers published in engineering are authored by women and the download impact for papers authored by women is higher than that of men. The analysis of success rates by FAPESP funding programmes (2016) shows high levels of successful applications for most categories of grants. FAPESP is of the view that is it not easy to disentangle the reasons for or causes of this presence of women in science in Brazil. The career structure and the organisational structure seem to be helping some women to succeed in science. In these times of growing scepticism about science, FAPESP would like to advance the science agenda to other areas in the production of knowledge through initiatives such as GenderlnSITE.

\section{Promoting Women Entrepreneurship Development via the Inclusion of Technology and Innovation (Dr Jill Sawers, Director: FemTech (Pty) Ltd)}

The McKinsey report 2017 said that if women were to occupy the same positions in the labour market as men, it would result in a $26 \%$ increase in the annual GDP of the world by 2025 , translating to $\$ 28$ trillion or 240 million jobs. An earlier study looking at Fortune 500 companies noted that companies with the highest representation of women at a senior 
management level actually offered a $35 \%$ higher return on investment. Teams led by women report more cohesion, cooperative learning and participative communication, and leaders operating with trust experience higher engagement levels with people working with them, which translates into better productivity, profitability and customer service and lower health care costs. These are among the reasons why gender inclusion matters.

The current situation is that in factor-driven economies roughly eight out of ten early-stage entrepreneurs are women who start businesses in the subsistence sector and out of necessity. Women in developing economies find themselves in highly competitive sectors and in the low-tech, low-growth sectors. In innovation-driven economies, roughly six out of ten start-ups are women. A study done in the US in 2011 found that women occupied less than $25 \%$ of STEM jobs and were earning less than $25 \%$ of their male counterparts.

A study conducted for Unilever (2017) interviewed more than 9000 men and women across eight markets and showed that the vast majority of not only men, but also women, believe that a man is the best choice to lead an important project. Both men and women overwhelmingly believe that men do not want women in top corporate positions.

In Sweden, approximately one-third of businesses are owned and run by women and it was noticed that they secured less than $18 \%$ of government venture capital (VC) funding. Harvard was asked to investigate the situation and their research showed that the male financiers' reliance on the stereotypical view that male entrepreneurs are successful and women are less successful translated into the actual funding results. Far more of the proposals submitted by women were rejected than those of men.

Some of the constraints facing women entrepreneurs in particular are:

- Very few women entrepreneurs have tertiary education or technical skills. They tend to start businesses in the services, crafts or agricultural sectors.

- Environmental constraints such as legal or institutional, financial constraints (due to no collateral in order to get funding for growth from financial institutions) and biases (conscious or unconscious).

- Women have fewer business networks and market opportunities. Women typically have many competing demands, transport and security issues that exclude them from mainstream business networks. Women have fewer, deep networks but not broad diverse networks required for spotting market opportunities. 
- Self-confidence is a very important part of women's entrepreneurship development. Women are often influenced by patriarchal societies that do not regard them as business leaders.

Findings from some programmes that Dr Sawers has been involved in are as follows:

- Mekong Women's Entrepreneurship Challenge

The programme followed a two-phased process that entailed selecting candidates and an innovation creativity and competitiveness workshop. This created a safe climate for the women and this encouraged them to share about their own business issues. It focused on helping them to think out of the box using certain tools and techniques. A selection of ten entrepreneurs progressed to the next programme. They were offered one-on-one coaching, successful role models (local women) shared their stories with them and they were given a small grant. The assessment at the end of the programme showed that the funding was the least important aspect of the programme and that offering cash to entrepreneurs developed a go-for-cash mentality rather than considering what the market wanted.

- FemTech Programme

This programme runs over six months in three series of 3-day workshops covering 17 modules that are very experiential. The women work in groups and apply theory to their business situation, and are given exercises to think creatively, spot the opportunities and differentiate themselves from being another 'need to' business. It was noticed that very few women entrepreneurs are in the technology space, and ICT and innovation were included in the programme as a way to help the businesses on the growth path. Specific modules give the women the opportunity to think about where they are on their journey and whether their business is aligned with their personal objectives. FemTech trains trainers to deliver the programme in the various countries.

- Women Innovators Network of the Caribbean Acceleration Programme

This is an InfoDev World Bank programme across 11 countries in the Caribbean. A group of facilitators was trained to deliver training to cohorts of participants, focusing on business themes and personal development themes. It was found that the latter gave the women a chance to reflect, develop and build their own resilience. The women are given mentors and experts talk to them on topics of relevance and interest to the women. 
Guiding design principles in all the programmes are:

- Mixed entrepreneurial support training programmes do not benefit female-led firms.

- Entrepreneurs learn best from trusted sources, such as their peers.

- Women prefer practical, relevant, hands-on learning.

It was found that the following contribute to the success of the programmes:

- A holistic approach that addresses both business and personal development, for building skills, self-esteem, confidence and resilience.

- A non-threatening space where entrepreneurs can share sensitive information with peers whom they can trust.

- A collaborative environment where entrepreneurs can experiment, reflect, adapt and apply.

- Developing the 'creativity muscle' for identifying opportunities.

- Encouraging participants to use technology to improve business processes and outputs via homework using tools.

- Facilitating a supportive network of business women.

- Upskilling local trainers and facilitators including building for sustainability.

\section{Women in Technology Businesses - Accessibility to Funding, Support and Markets: TIA Case Studies (Dr Anitha Ramsuran, Manager: Innovation for Inclusive Development, TIA)}

TIA does not have a special focus on women, although it does need to produce data in relation to support to women and previously disadvantaged individuals. TIA offers three funds: seed funding, technology development funding and commercialisation support funding, which are measured according to technology readiness levels. Applications are assessed for market readiness, as well as business readiness. TIA also offers a number of supporting programmes, namely:

- Technology Innovation Cluster Programmes.

- Technology Stations Programme.

- Technology Platforms Programme.

- Innovation Skills Programme.

- Youth Technology Innovation Programme.

- Global CleanTech Innovation Programme. 
A small empirical study was done to look at TIA's investment in terms of women innovators and the support it provides for women. The following questions were posed to TIA investees in the period 2012 to 2017:

- What are the unique traits of women who have developed innovative technologies?

- What informs their choices of solutions they develop?

- What challenges must they overcome to succeed in bringing technologies to market?

- What support is available to women-owned small, micro and medium enterprises (SMMEs) in terms of funding, innovation, support and access to markets?

The study found that TIA had approximately 82 investments, with 12 investments through the Technology Development Fund (above R1m each), 50 investments through the TIA Seed Fund (up to R500K/R650K), one investment in the Youth Technology Innovation Programme $(\mathrm{R} 1 \mathrm{~m})$, one investment in the Technology Platform Programme and 18 investments through the Innovation Skills Programme and Global Cleantechnology Innovation Programme.

The following case studies exemplify the kind of innovation that is coming from innovators in South Africa:

- Artificial Mind Engine (AME), which is a cognitive analytics software platform suitable for the Big Data and Internet of Things (IOT) markets, by Anet Potgieter. The technology speaks to SDGs 9 and 11 .

- Homestead Biodigester, by Rasmene Zanele, using biomass to create bioenergy. The technology speaks to SDGs 7 and 11.

- Olive pomace extracts for cosmetic applications by Nicole Forster. The technology speaks to SDGs 3 and 13.

- The Para-Tube (a toilet system built onto a wheelchair) by Portia Mavhungu. The prototype is being developed and the technology has not yet reached the market. The technology speaks to SDG 3 in particular.

- Coor-Tech Textile Waste Treatment Reactor by Prof Veruscha Fester. The technology speaks to SDGs 11 and 13.

The female innovators of the above case studies indicated that the unique traits that made them successful innovators and informed their choice of technology were:

- Their ability to persuade others to believe in their ideas.

- Their focus on setting and reaching goals. 
- Their talent for team building.

- Their courage to break the rules.

- Their eagerness to learn and empower other people.

- Their desire to uplift others.

- Their ability to apply their skills (interpersonal and communication) in bringing about innovation.

- Their need to bring about change, making a different and touching people's lives in a different way in communities.

- Their creativity, dedication, motivation, determination and intuition.

- Their gift as care givers.

The study highlighted the following challenges in bringing the solutions to market:

- Processes are unknown.

- Regulatory delays and processes that do not run according to plan.

- The lack of published information or people who have gone through the process before.

- The stereotyping of women in relation to their technological abilities and knowledge.

- The scepticism of the scientific world, funding, the local regulatory system.

- The lack of skills, expertise and service delivery in the manufacturing industry.

- The extended time period taken to bring the concept to demonstration level.

- Persuading industry to invest in a new way of doing things.

- Finding the funds to bring the technology from concept to demonstration.

- Difficulties in being able to explore market opportunities.

The study identified the following experiences of innovators in relation to funding and innovation support:

- The need for $\mathrm{VC}$ to bring processes and promising technologies to market.

- Government support systems are wonderful.

- The TIA funding system is helpful in that it forces inventors to go for commercialisation and innovation training. 
- The system is fair. There is no differentiation between male and female applicants.

- TIA provides the necessary support regarding funding and innovation throughout the drawn out and difficult process.

- The CleanTech Accelerator Programme is very helpful in that it provides access to mentorship and other business support needed to establish a start-up.

In terms of access to markets, respondents indicated that:

- The Technology Transfer Offices (TTO) assisted in reaching out to markets.

- Minimal support was received and there was a lack of a sense of urgency on the side of TIA.

- Mistrust in sharing guidance and experiences are huge barriers to market entry.

- Some innovators are piggy-backing on existing markets.

- Support received via TIA through entrepreneurship programmes and pitches to international technology agencies (Switzerland, Israel and France) were helpful.

- Support received via domain expert business partners to access new market segments such as health, video-surveillance, security/ perimeter access control, mapping and telematics.

\section{QUESTIONS AND COMMENTS}

Ms Kisjes: How are the top-down approaches mentioned by Prof Schraudner incentivised?

Response, Prof Schraudner: The size of the grant is an important part of the incentive system for professors at universities. The idea of addressing more societal needs has become more and more important. Within the Excellence Initiative, which is really changing the research system, universities are looking for good indicators to find incentives for these activities. This is something new. Publications and student production are no longer the only incentives.

Prof Malcom: Prof Schraudner's one slide showed changes in policies that had occurred in different kinds of funding agencies. What is the next thing that needs to be added to the criterion for funding that could make the greatest difference in terms of the way that all of this gets managed? 
Response, Prof Schraudner: It will take time to change the mindset. In Germany, there are some universities that try to focus on pure research only. More and more are opening. The new Excellence Initiative, especially the 'third mission' aspect of addressing societal needs in and with society is the most interesting way of differentiating the universities. I see a clear change in that direction. Engineers and social scientists are coming closer together.

Prof Malcom: Would you recommend that more transdisciplinary structures or centres that allow the different areas to come together to address societal needs are needed?

Response, Prof Schraudner: I do not think it is helpful to have a specific centre. I believe that people can interact on specific issues. Perhaps it is helpful to have another way to write proposals that provides for participants working together. This is more efficient. In Germany, there is a new institute for digitalisation with engineers on the one hand and social scientists on the other hand. I am not sure that this is more efficient than having five big projects where people from different disciplines can come together from all over the country to work on the projects.

Prof Watanabe: We heard that in Brazil the ratio of female-to-male researchers is around 50:50. Are there fewer female researchers in industry than in academia?

Response, Prof Almeida: Women earn less than men in Brazil in all fields. Representation of women is lower in all fields and there is a huge gap in engineering and natural science. Men and women with PhDs are not found in industry and they are not as connected with industry as they should be, because industry is a good environment for innovation. An academic career seems to protect women from situations found in Europe, for example. They are more comfortable in building a career in academia, and because they can afford to pay others for child care, they have a huge advantage in the job market.

Prof Thomson: The women innovators mentioned by Dr Ramsuran spoke about the long and difficult process that TIA puts them through. Can you explain this?

Response, Dr Ramsuran: I do not think this is a good thing. TIA has recently improved its processes. The process is not as long as it used to be. We are trying to reduce the time from application to funding to four months instead of two years. We are working very hard to keep improving our processes. 
Prof Malcom: Does TIA have a recognition programme for the very successful entities that receive funding from TIA? In the US we have the small business innovation research funding and regular awards are given in recognition. This shines a light on the ones that are successful so that they can become role models.

Response, Dr Ramsuran: Some specific TIA programmes have their awards and this produces role models. Broader awards are being considered. TIA is a new entity and a lot of processes are still being refined.

Prof Malcom: Looking at the notion that many elements are important and that money is not necessarily the most important, in the US, questions about harassment experienced by women have been raised. Is there a way to identify this and are there laws in place to ensure that individuals who may engage in such behaviour can be punished?

Response, Dr Sawers: There are laws in South Africa to take on cases of harassment. Even though our experience is that in the early stages funding is not necessarily the most important aspect, growing an enterprise will require growth funding. This topic is addressed through mentorship. Mentors are screened by the facilitators or trainers, and relationships will suffer if untoward or inappropriate behaviour is evident. Where funders are brought in, they are people from within a reference group that we know, and there are no issues of harassment.

Response, Dr Ramsuran: TIA has built in a right to refusal clause, which allows TIA to first right to fund the investees further or to refuse funding. TIA facilitates relationships with follow-up funders and the VC community.

Response, Prof Almeida: There has been a lot of investment in mentoring and in making sure that the projects are closely followed by university officials. We also have an innovation in small business programme, which is overseen by university officials. Brazil does have laws, but there are complaints that they are not sufficient to deal with the situation.

Unknown person: Looking at the current environment, a number of entrepreneurs complain that the instruments being used to evaluate them are the same as those being used by commercial banks and do not apply to start-ups.

The presentations did not reflect the aspects of the corporate world that come into play. In some cases, it is corporates that sponsor the inventors to run with the technology until it has been commercialised. 
What percentage do you allow for these scientists to claim as salaries? In some cases, funders refuse to fund salaries, while the investors are cash strapped.

Response, Dr Ramsuran: You are correct that the TIA application looks the same as those for cross-funders, but the difference is in terms of the way the deed is structured and the type of return requested. TIA operates mainly on a grants system with a certain percentage of royalty upon commercialised. TIA does cater for salaries.

Response, Dr Sawers: There is an example from an incubator in Tanzania where salaries were an issue for the very early stage start-ups. They negotiated with a big company to project manage a programme to which the entrepreneurs contributed and were paid a stipend.

Response, Prof Almeida: In Brazil, projects involve a relationship between the entrepreneurs and the scientists. The scientists receive grants but the entrepreneurs do not receive any salaries.

Mr Perkins: Dr Sawers mentioned that quite often networks were established for support amongst the entrepreneurs. What are the conditions that enable that?

Response, Dr Sawers: Data collected from some of our programmes showed that the most important benefit listed by far the majority of participants was about learning from peers and being part of a network of support. This was enabled through the methodology applied from day one. An environment is created where there are no competing businesses and where there is trust. The support networks often start with WhatsApp or Facebook groups, and develop a life of their own with individuals taking the lead.

Mr Perkins: The statistics of what FAPESP has been able to achieve are very impressive and the commitment of $1 \%$ of the budget is substantial. How did this happen?

Response, Prof Almeida: A scientist who was part of the state parliament put forward the idea of an agency to develop research in the state. It happened during the time that the FAPESP was being established. By the end of the 1960s there was a provision to include $1 \%$ as a law in the state constitution. This was mainly a legislative initiative started by a scientist who was a politician and supported by important politicians. The effects of this law were only felt much later, towards the end of the recession when growth began. Since the 1990s, FAPESP has been 
actively encouraging other states to implement research funding organisations. There is always pressure to show that what FAPESP does is very important for society.

\section{PANEL 4}

\section{Measuring Women's Participation in International Patenting (Prof Bruno Le Feuvre, Statistical Analyst, IP Statistics Section, World Intellectual Property Organisation Geneva 20 (WIPO) (Via Skype))}

Prof Le Feuvre presented recent work done by WIPO on measuring women's participation in international patenting. The original idea for the study came from the goal set by the UN to achieve gender equality. One of the ways that the Economics and Statistics Division of WIPO could contribute to this goal was to identify women's participation in the filing of intellectual property (IP), looking at women's participation in innovation more broadly speaking. However, this was not easy because a patent application does not indicate the gender of the applicant. The methodology developed is detailed in the publication, Identifying the Gender of PCT Inventors, which is freely available and has to do with the creation of a World Gender-Name Dictionary comprising 6.2 million names that are valid in 182 countries.

The Patent Co-operation Treaty (PCT) international patent application system is administered by WIPO and represents about $60 \%$ of all patent applications filed in the world. The gender of about $96 \%$ of all inventors listed in the PCT international patent applications is identified by using this dictionary. WIPO has mainstream statistics by gender, which have become part of the regular reporting on the PCT System in the PCT Yearly Review. Data from this review show that:

- The number of PCT applications where at least one woman is named has sharply increased in recent years and the number of PCT applications with women inventors has increased three to four times from 2002 to 2016.

- Women's share of applications has increased from about $20 \%$ in 2002 to $30 \%$ in 2016 . This shows that the number of PCT applications with women inventors is increasing faster that the overall number of PCT applications, even though the gender gap remains high.

- The share of PCT applications with women inventors by geographical region (2000-2016) shows that Africa has the lowest share (19.7\%) and Latin America, North America and Asia have between $30 \%$ and $33.7 \%$ of the share. 
- The figures by country show that women inventors represent notably high shares of PCT applications in the Republic of Korea and China, taking into account that the total number of applications from these countries has grown substantially.

- One of the reasons for the difference in the share across countries could be the field of technology of the applications. The figures show that women inventors are represented in high shares of PCT applications relating to life science technologies, particularly biotechnology and pharmaceuticals. This can be explained by the fact that more women work in the life sciences.

This work was a first step towards identifying women's participation in innovation and further research is necessary. Some statistics by gender for selected countries, looking at national patent applications, will be published in December 2017. Studies will be expanded to all the intellectual property rights, for example, industrial designs or trademarks, and the number of sources for the dictionary will be increased. Gender indicators will have to be refined in order to gain a better understanding of women's participation in IP filings and how this changes over time.

All the reports are available on www.wipo.int/ipstats and WIPO's Economic and Statistics Division can be contacted at ipstats.mail@ wipo.int.

\section{A Newe Way by Her Invented (Amy Everard, 1637) (Prof Anne Grobler, Director: DST/North-West University (NWU) Preclinical Platform, NWU)}

The Preclinical Platform is a national platform and a joint initiative between the DST and the NWU. It came about when medicines were being developed, but acceptable and compliant facilities needed to do animal studies could not be found in this country and appropriate facilities had to be established. A whole new ecosystem in terms of drug development was created as a service to the country. The platform became self-sustainable after the initial five years and is now also used to do drug evaluations for international companies. While the platform is used to progress inventions, it is also been used to embark on business opportunities, such as establishing industries in the local communities to support the work done in the platform.

Innovation is something new. It is both a concept and an activity and enables a transformative approach to economic growth and society's challenges. Innovation relates directly to the SDGs. A system of innovation should contain all elements and relationships required for the production, absorption, diffusion and use of new and economically 
useful knowledge. Patenting requires innovation to be novel and to be usable. Knowledge generation and knowledge discovery are two different things. Innovation is knowledge discovery and universities generate knowledge. Perhaps it is necessary to move away from the idea that innovation needs to take place in academia and understand that there is also grassroots innovation and citizen science.

Historical efforts to increase the participation and impact of women in science have produced limited results at the advanced level of research and development (R\&D) and innovation production. The lessons learnt from these efforts and about what worked and what did not work have not been properly evaluated. This aspect ought to be addressed in South Africa. Gender equality should not result in parallel systems to foster the research and innovation system, but should be systemic. It is known that women are really good at intuitive science, mathematics and functional design and they should leverage these strengths.

Prof Grobler is well placed to give advice on gender innovation, and told her story. She is a recognised inventor with patents and has developed products that are on the market. When she started her career she could not get a housing allowance and she could not register her son on her medical aid because she is a woman. There are huge differences between the past and the current situations in terms of rights. In her earlier career, she was known as the idea generator (not an inventor) and did not like writing papers. The situation has changed substantially over the last decade and although tremendous progress has been made in South Africa, much still needs to be corrected. Women want fairness and not an advantage above their male colleagues.

Prof Grobler presented several examples of innovations coming from her (and the platform's) work that have led to patents in various countries across the globe, and told of her experience particularly with regard to funding, IP, strategy, exploitation and other uniqueness factors, and progress made with regard to gender equality in science since the early days of her career. Translation of research and innovation for social impact is important and new national research institutes in key strategic priority areas ought to be considered.

In terms of outputs to date, the platform has delivered 90 postgraduate students in different universities completing PhDs and MScs on Pheroid technology, as well as numerous papers and patents, three companies have been established and 18 jobs in industry have been created. Approximately 90 tons of an agricultural product are being produced 
every year using green technology and distributed across the world. More products are being developed using the same technology. This product has enriched farmers in South Africa and China by about R2 billion over the years and has benefited food security.

In order to change the model, scope and context for women, it is necessary to look at all of the players: public research institutions, funding bodies, state-owned entities, industry and regulators. A model has been set up with multiple structures, providing for a national platform and incubator, a not-for-profit company and for-profit companies earning royalties and manufacturing different products. The regulatory space in South Africa is problematic as it constrains STI and needs to be appropriate for the knowledge-based economy of the 21 st century.

A quote by Henri Poincare states that "It is through science that we prove, but through intuition that we discover". Women have very strong intuition.

\section{Contribution to Greater Equity in the Attainment of the SDGs (Mrs Berenice Marais, Executive, Innovation Enabling and Support Programmes, TIA)}

Mrs Marais' presentation focused on her many years' experience in working in the STI arena, from the South African perspective and as the African regional representative for the World Association for Industrial Technology and Research Organisations (WAITRO), which has a membership of about $90 \%$ of Africa's research, development and innovation (RD\&l) institutions, or about 52 African institutions. She reflected on how to contribute to greater equity through looking at what is happening in the South African environment and the African continent.

South Africa has a very strong policy environment and visible funded programmes that are focused on gender initiatives, which are well supported and well initiated from both the public and private sectors. South Africa also has women-led initiatives either in the public or private sector. There is a very strong advocacy for women, lots of visible programmes and there is funding available. This country has high levels of international engagement and representivity across the world. The Head of UN Women was the Deputy President of South Africa from 2005 to 2008, and women are well represented in leadership positions throughout the country, also in Cabinet and in Parliament. National Treasury and government departments have key performance indicators that measure quantitative results, although they do not 
necessarily measure qualitative impact on the ground. However, there is an over-reliance on reporting and institutions are spending more and more time reporting than being involved in activities on the ground. In addition, there are high levels of community engagement of women in South Africa. The voice of women is very strong, but unfortunately most of the debates are extremely polarising and dominated by gender problems, severe violence, lack of access to economics and regulatory issues.

The key challenges in South Africa and across Africa are:

- Many of the programmes in the National Innovation System count the number of women scientists. Although this is important, there are not enough rewards and acknowledgements for women who are managers of science and enablers in the STI space and who face management constraints and look to improvements to institutional processes. The TTOs across the country deal with many frustrated scientists who have left science and gone into management as a means to rid the system of impediments. The efforts are noble and need to be recognised.

- Resource constraints, which lead to a lack of access. There is heavy community-based activism, a lack of services and low public service delivery.

- The very low levels of applications from the disenfranchised youth and women. This is problematic, particularly as more than $60 \%$ of the youth are unemployed and unskilled. The kinds of innovation applications that TIA receives from that disenfranchised sector are mostly social innovations dominated by indigenous knowledge, health, cosmetics and agriculture. They have low levels of innovation and are of low value.

Lessons learnt are:

- There is a need for more recognition for science enabling and management actions.

- There is a need for more selective calls for women in science that include social inclusivity criteria.

- The debate about gender in South Africa is extremely politicised and used as a political tool. It is not projected enough as a technology enhancing issue.

- Gender policy and programmatic interventions must involve active role modelling, culture change, supportive actions and funding instruments, specifically in the everyday context of South Africa. 
Some questions to be raised are:

- How will the constraining and supportive actions for gender-based initiatives be addressed?

- Where is the voice of the youth? The youth are pleading for gender neutrality. This has implications for the discussions on gender and innovation.

- Where are the inventions by women? These stories need to be profiled more vividly and their impact on quality of life and the revenue aspect need to be made known.

- Using social media, how do we position our conversations and our programmes on youth and gender differently? It is necessary to consider the issue of gender neutrality.

\section{QUESTIONS AND COMMENTS}

Prof Thomson: Prof Le Feuvre, in your future work, would you include plant breeders' rights?

Response, Prof Le Feuvre: No. The next stage will be to identify the gender participation in the Hague System for industrial designs. The challenge we face is to have a database of intellectual property rights that allows us to extract statistics from the data.

Prof Thomson: At the national level, some countries do investigative work on their patents and others accept them as they are. Do you differentiate between that?

Response, Prof Le Feuvre: For patents at national level, we use a database that contains published applications and we could certainly focus on countries where the data sets are of good quality. Usually, it is the offices that do formal examination of patent applications.

Prof Buitendijk: Can you differentiate between women who are leaders in the patent applications from the rest of the group (in respect of the number of women inventors in international patent applications)?

Response, Prof Le Feuvre: This would be extremely interesting, but sadly not. This is because there is no order in the list of names of the inventors/ applicants. Usually the applicant in a patent application is a company and the company will list inventors in no particular order, so it cannot be assumed that the first name of the list is the main contributor. 
Prof Malcom: Different institutions have different rules about whether or not graduate students or postdocs can be included on applications. Given the fact that the make-up of the senior population differs from that of the student population, which tends to have more women, would there be any way to capture any of this information?

Response, Prof Le Feuvre: We could not analyse a lot because we rely on the information provided in patent applications, which have a limited number of variables. The study that we do and the dictionary we have produced are shared with researchers to do further research. There is a lot that can be expanded and improved by using all the databases.

Prof Watanabe: Some researchers in Japan analysed the economic value of patents. They divided into two groups: one group was only males and the other was mixed male and females. The economic value and advantages of the two groups were compared and the value of a mixed team of inventors was seen to be higher.

Response, Prof Le Feuvre: When international patents are not yet granted and before formal examinations, it is difficult to tell the quality of the application. There is a lot that we can do especially in respect of national patents, where we will be able to dig further and compare the different profiles of applications.

Prof Malcom: AAAS has a programme called the AAAS-Lemelson invention ambassadors programme. We select people who are recognised for their invention and arrange opportunities for them to go out and speak and become more visible in representing invention and innovation and the impact it has on society. The classes of individuals are very diverse. One thing we hear from them is that they get very little recognition within the institution and that patents do not count towards tenure. This makes it very difficult to justify why one would do all the work. Is it an unwritten rule of the academy that patents are not given the same attention within the overall structure of recognition?

Response, Prof Grobler: Yes, I think that is true. Even in the National Research Foundation (NRF) evaluations, patents have only become noticeable in the last three years. Prior to that, there was no space on the forms to include patents on one's CV. This has started to change but still does not carry the same weight as publications. It is much more difficult to get patents granted in different countries than to publish. I have spoken about my inventions in a number of countries but not in South Africa. 
Prof Malcom: Does it carry weight in terms of Membership of ASSAf and in academia?

Response, Prof Diab: ASSAf Membership application forms ask for top publications and not for patents, and the assessment is done through an election procedure. Membership of ASSAf requires excellence in science, which can be either through publications or through patents, but it is for society. Certainly patents have an even greater contribution in this regard. Perhaps this is something we need to think about. In academia, there is an opportunity to include patents. The Centres of Excellence are pressured to get patents and are judged on the number of patents they have. This is not yet the case for individual researchers.

Response, Prof Thomson: By the same token as we ask for citations in publications we need to know if the patents have been taken up.

Response, Prof Globler: Patents, by their academic value, need to count because they do have substantial scientific integrity. However, there needs to be a second evaluation system for what has materialised from the patents and what the impact has been. Two different metrics are required.

Dr Comins: Both Prof Thomson and Prof Grobler have come across strongly as having changed the culture of their departments at universities, with the number of students increasing probably because of the culture. How does that position you in terms of other members of staff in the department?

Response, Prof Thomson: This has not been a problem. In our department, everybody is strong in different fields and they delight in each other's successes. I found that other departments were amazed at the number of black students (particularly from other African countries) I attracted. This happened because of the field I am in and because many of those students wanted to make a difference in their own countries.

Response, Prof Grobler: It is not unproblematic in my case. What has happened is that the department attracted strong women, although not by virtue of selection. Of our postgraduate cohort, $78 \%$ are female students and they are excellent. It is possible that by changing the culture, more women are attracted. Role modelling success also helps.

Prof Malcom: I find that there are instances for providing opportunities for younger and younger students to think about invention and entrepreneurship through 'making'. Has offering maker spaces to students who are still at school taken off in South Africa? 
Response, Prof Grobler: We have engineering students who became interested in one of our inventions and built the hardware necessary for the invention to work. This was very satisfying to see. I believe that this country should move form a consumer country to a production country.

Mrs Marais: TIA has a Youth Technology Innovation Programme, which starts at 18. Because of the historical lack of access, we focus on the disenfranchised youth. Most of the applications are not students that have graduated and are probably those that cannot make it into these programmes and come out of community colleges and high school but are doing nothing and looking for innovations in rural and low-tech environments. We have tried to focus our efforts in teaching innovation skills and helping them to write a proposal, put up a website, do a pitch, meet an investor and develop a prototype.

Prof Diab: I want to explore the remark that youth are pleading for gender neutrality. What is driving this?

Mrs Marais: The plea for gender neutrality is definitely here already and I think it is global. For the youth of today it is all about human rights and freedom of expression of these rights. When the youth talk about equality they do not talk about gender differences.

Prof Grobler: I resent the fact that I should receive special dispensation for being female. I view myself as equal to a man. The fact that certain things are unfair towards women comes from the originators of the problem.

Mr Perkins: From the perspective of the UK and a lot of the discussions among children in my circle, gender issues do not need to be stressed. However, globally it is clear that gender is still an issue that has a material impact of particular groups. We have to be careful not to conflate these two things.

Prof Buitendijk: This is a fascinating discussion. Gender is much more dominant than it used to be. Young men are also feeling the pressures of how they need to be as men. We should not forget that equality, less emphasis on the differences and less stereotyping between genders will also make young men much happier. Gender-based violence is largely due to the roles that society forces on boys and girls. A lot of research that looks at differences pertaining to education between boys and girls shows that if you treat them both as equal in the classroom and if there is less gender behaviour and gender teaching, boys' grades 
will go up. Many young children do not like gender conformity and behaviour expected from them.

Prof Malcom: In the US, feminist thought and discourse is increasing.

Dr Sawers: We found that in the entrepreneurship space there is the definite need to create an environment where the women can develop and grow. We have offered sessions on women in a men's world and men in a women's world. The men were fascinated with the discussions and did not realise that the women were facing these issues.

\section{DAY 2 (MODERATOR: ALICE ABREU)}

\section{PANEL 5}

\section{Gendered Innovations (Ms Anne Githuku-Shongwe, Representative for East and South African Region, UN Women)}

The role of UN Women has to do with gender equality and women's empowerment. Data in relation to SDG 5, focusing on gender equality, about what is known about the current situation with regard to gender equality shows that:

- The global labour force gender participation gap is estimated in the global economy at about $\$ 1.6$ trillion. The average pay gap between men and women across the world is $73 \%$.

- In terms of gender violence in South Africa, research done very recently in Diepsloot (a peri-urban community in Johannesburg) shows that $64 \%$ of all the men in the study admitted to having sexually assaulted (at least) one woman in the last six months.

- $34-35 \%$ of the global economy's GDP comes from public procurement, but only $1 \%$ of that goes to women. This figure is interesting in the context of the South African economy where about R635 billion goes into public procurement, $6-9 \%$ of which goes to women.

When looking at the issues of gendered innovations, the starting point is already unequal. It is assumed that life is equal, but it is not organised equally. Research is viewed as equal but it is not equal. Stereotypes are embedded. The ability to put on the gender lens is the first barrier to be crossed when doing research. Some examples of the work being done at UN Women to try to resolve the big issues relating to gender are: 
- The public transport system, as a public space, is one of the biggest drivers of violence in South Africa. An initiative was started in Cape Town some years ago as part of the global programme called Safer Cities, working in one of the more violent communities, particularly in terms of sexual violence, and looking at how to create an environment that is safer for women. The community helped identify hotspots, one of which was bus routes with stops in close proximity to taverns. The routes were changed to bypass these vulnerable spaces. The city realised that the gender lens perspective has to be considered when planning public transport routes.

- A big procurement initiative to move procurement opportunity and markets into women's hands. It was found that almost $90 \%$ of the chief procurement officers in companies are men with 'old boys' networks' that exclude women. An enterprise solution called Buy from Women was created to address this issue. The platform is designed to match procurement opportunities in government and corporates with women entrepreneurs. It was used in Rwanda to empower women farming cooperatives to understand their yield and productivity and their needs, and to connect them with orders for their produce. The platform has helped 3500 women farmers to completely change the economy and break the barriers that existed to them participating in the market.

There are many opportunities to walk alongside women in addressing gender inequalities. It is most important to recognise and understand what policies are required, and when adding the gender lens, it is possible to influence how people innovate and what they create as if women matter. The SDG challenges present the opportunity for women to start changing the story. Design thinking needs to be embedded as if women matter. This has been embraced at UN Women. Climate change is a big area that UN Women is working in. Incredible innovations have been seen in South Africa with regard to climate change, where women have come together as massive cooperatives in this time of drought and use recycled plastic bags to grow excessive amounts of food for their families and communities. UN Women is partnering with them to supply the World Food Programme with food to take to other communities.

Ms Githuku-Shongwe founded a digital enterprise creating gamification learning solutions and shared some of her experiences as a woman entrepreneur in the area of gender and innovation. 


\section{The Transition from Research/Invention to Innovation - Is it Gender Neutral? (Dr Neville Comins, Independent Consultant)}

The transition from research/invention to innovation is often underestimated from a researcher's point of view, but if there is to be a change in activity this will have to be unpacked. South Africa spends approximately $\mathrm{R} 15$ billion a year on research, but this does not present a bright picture in terms of how it has translated into the economy over the last 20 years. If there is to be more of the same, then the SDGs will remain a dream. In order to be able to achieve the SDGs, the need to change things will have to be taken seriously.

A very useful paper written by Jeffrey $D$ Sachs analysed the transition for the Millennium Development Goals (MDGs) to the SDGs, highlighting some of the serious changes needed in respect of:

- Technological and social change.

- Mobilisation of global knowledge operating across many sectors and regions.

- Global problem-solving networks for sustainable development.

- Pathways to sustainable development will be identified through a highly energised era of networked problem solving, engaging the world's universities, businesses, non-governmental organisations (NGOs), governments and young people.

- The involvement of young people, as they will become the experts and leaders of a new and profoundly challenging era.

- The lifeblood of the MDGs and the SDGs, which should be data that are accurate, timely, and available to managers, policymakers, and the public.

In South Africa, the driving concept since 1996 has been a NSI stimulated by enhanced research and underscored by a campaign to grow the number of PhD graduates. Various studies by the Organisation for Economic Co-operation and Development (OECD) and a Ministerial Review in 2012 indicated that the NSI remains very fragmented and this affects its ability to deliver. Given its capacity, South Africa must keep abreast of the global knowledge in critical fields for the economy. Findings from the 2010 ASSAf review of PhD programmes in South Africa using 2007 data showed that women's participation in PhD programmes was strong in health and social sciences, and $15 \%$ of women graduates studied engineering sciences, materials and technology, but only $36 \%$ of them were South African nationals. This is concerning in the light of the expertise and skills needed to solve the SDGs. The reasons given by the cohort of doctoral graduates for 
having studied a PhD raises concerns that their focus is inward and personal and they are not driven by a desire to implement their work in the real world. This inward looking culture could become inbred, particularly as more than $50 \%$ of the PhD graduates find employment in the higher education sector. The report highlights industry employers' views that the $\mathrm{PhD}$ graduates lack exposure to international expertise, theories and debates and do not have 'real world' relevance.

Many of the accepted challenges facing South Africa and a number of similar countries are mirrored in the SDGs. The needs and solutions to the SDGs require new ways of doing things, which may include technology but certainly includes changes in societal behavior. If the knowledge generating structures do not strive to provide this or to assimilate world experience, more of the same will continue. It is necessary to look at better strategies to identify the needs and the solutions and to have much more co-operation and collaboration, networks and partnerships, sharing and learning, and innovation. The characteristics of successful women in business (from an article by Sharon Hadary) correlate well with the latter list of what needs to be done. This gender debate is much more to do with foundation material that it is a superficial 'fix-it'.

Innovation is not invention. It is the process of taking ideas right through to the market or application in society. It must have customers and markets and it must demonstrate an economic output or a social impact. In the current era, innovations are often associated with new technology developments, but this is not a necessary condition. Innovation in reality is strongly linked to business and industry or an enduser.

Most South African graduates recognise the role of research to generate new knowledge and the measures and outputs have recently been expanded to consider IP as important, yet far too little is known about how to bring such knowledge or IP to successful innovations, which is the key to achieving the SDGs. In addition, there is minimal education on the complexity of taking ideas/inventions to market for societal use. There is an intellectual and physical gap between research (linear thinking) and innovation (needs and market driven). The likelihood of research meeting the need and leading to successful innovation is tenuous at best, and the linear model is basically flawed. Going from research and invention through to the economy requires entrepreneurial behaviour. The innovation cycle is complicated and research is not an isolated element, but a highly interconnected set of nodes. Connection with others is crucial. The problem is that licensing 
out the IP breaks the bond between the research capacity and the end point.

There are also gaps in the approach to innovation. Graduates only become exposed to innovation once they move an idea to the TTO and entrepreneurship is not a requirement for graduate students. Although there are increasing numbers of women graduates, involvement with successful innovations remains limited. This raises questions about whether women are encouraged to think about innovation (perhaps it is a male preserve) and whether women graduates reach a life stage where family matters become paramount. More information is needed to understand this.

In conclusion:

- An analysis to define needs/problems and formulating of possible solutions will be enhanced by full gender participation.

- In terms of the SDGs (and the NDP), new approaches are needed to determine what should be established, with gender parity as a requirement, prior to any call for research proposals.

- Regarding gender balance as being about 'pinkifying' projects to satisfy a quota or 'tick in a box' exercise must be avoided.

- The gender mix can clearly bring about new approaches with enhanced networking and collaboration skills to stimulate reduction of fragmentation in the NSI.

- There is a desperate need to expose all STEM and social science graduate students, especially women, to entrepreneurial concepts and innovation stages in order to enhance innovation from research. This will increase awareness of the complexity of implementation and allow key relationships to be established during the research phase.

\section{Gender and Entrepreneurship - Potential Benefits for Investment in Innovations (Dr Chamunorwa Togo, General Manager: Innovation and Industry Development, The Innovation Hub Management Company)}

It is important to develop and build confidence in young women, and support them in what they want to do and in what they do best.

The Innovation Hub is an initiative of the Gauteng Provincial Government. Its work is to turn ideas into money by commercialising research products focusing on three main sectors: Bioeconomy, Green Economy and Smart Industries, encompassing ICTs. The majority of the Hub's Bioeconomy companies are female-headed. As exemplified by 
some senior female academics, women have specific skills that enable them to convert university departments into warm, welcoming and habitable places of work for all. It is not a secret that females excel in the life sciences.

Successful innovation and entrepreneurship require a group effort. TIA focuses on the technical aspects of products of research to ensure that they are ready for commercialisation. The Innovation Hub works with TIA to impart business skills and business mentorship to innovators. It looks for funding from the Industrial Development Corporation (IDC) for the upscaling of technologies and approaches the Department of Trade and Industry to source immovable infrastructure. The Provincial Government provides incentives for business development.

In order to address gender and innovation, it is important to start by changing some of the fundamental aspects, such as:

- Better communication and more visibility for initiatives led by women.

- Reviewing the ratings and how universities operate in terms of the promotion system. The current system focuses on publications and producing graduates, but neglects entrepreneurship. The university system must dovetail and have synergy with industry and ensure that it produces what the environment requires and graduates that are relevant to society.

- Challenging some societal norms. Maternity leave as well as paternity leave need to become the norm. Mothers need to have time with their young children as this, among other things, will improve children's (particularly girls') confidence levels.

- Natural leadership skills of women need to be recognised and used to the benefit of society. Most people measure success by looking at how many people they are in charge of, but success should be measured by the number of people they are walking with and have brought alongside them.

Sustainability lies at the heart of the female. The saying, "behind every man is a women" should be changed to "at the centre of every successful initiative, is a women".

\section{Gender and Scientific Research: Perspectives from a Gender Audit of Kenya's Climate Change Policies (Dr Winnie Khaemba, Research Fellow, African Centre for Technology Studies (ACTS))}

The gender audit of Kenya's Climate Change Policies (i.e. the Climate Change Act of 2016 and former Climate Change Policy of 2016) aimed 
to identify key strengths, determine weaknesses and provide key recommendations on how gender can be better incorporated into these policies. The main issues looked at had to do with the inadequate gender responsiveness in policies and policymaking processes, and limited research undertaken to understand how men and women are adapting to climate change, mitigating emissions or coping with loss and damage as a result of climate change. The audit was driven by the UN Framework Convention on Climate Change and a document called the Lima Work Plan on Gender and its Gender Action Plan drafted in 2017. The method involved the following:

- A review of the legislation documents, most importantly the Kenya Climate Change Act and the draft climate change policy.

- A survey questionnaire deployed to relevant stakeholders.

- Ranking of indicators.

- An analysis of the identified indicators.

- A report.

The indicators identified were:

- Scope and definition.

- Decision-making and representation.

- Participation.

- Inclusion of gender targets.

- Measuring.

- M\&E (monitoring and evaluation) framework.

- Financial mechanisms (institutional and enabling framework).

- Institutional mechanisms (institutional framework).

- Dissemination of information (including capacity building).

- Expertise and human resources (in respect of gender in climate change).

Using the indicators, some of the strengths of the policies were found to be:

- Gender objectives exist and are defined in the policies.

- The policies outline a financing mechanism known as the Climate Change Fund, which is required to ensure gender responsiveness.

- The gender objectives guarantee equitable representation on the Climate Change Council.

The main weaknesses were identified as:

- The lack of specificity in the gender objectives and goals. 
- Gender-specific targets are not explicitly outlined, providing a challenge for implementation and reporting.

- The lack of strong linkages between climate change and disaster risk reduction. This is especially a concern because climateinduced disasters widen the gender gap and further exacerbate impacts on women.

- Inadequate focus on capacity building and training to build expertise and create awareness on gender and promote gender equality as a means of reducing vulnerability to climate change.

- The lack of a clear role for the National Gender and Equality Commission, which is mandated to monitor and report on gender.

\section{QUESTIONS AND COMMENTS}

Prof Schiebinger: Is the work done to grow food in plastic bags written up and is it possible to get a citation so that we can read the story?

Response, Ms Githuku-Shongwe: There is research going on by Gender CC, an organisation that does gender climate change work. The work should be available by the end of this year, and will be circulated to the participants of this workshop.

Mr Perkins: Ms Githuku-Shongwe, what key lessons did you learn from your experience of being a tech-entrepreneur?

Response, Ms Githuku-Shongwe: I did not set out to be a techentrepreneur. I wanted to solve a specific problem that happens to use technology. The lesson is that technology is really just the tool for the solution and it has to be kept as such. One should not get caught up in the technology itself, but keep the environment in mind. One of the biggest challenges was to do this alone.

Prof Malcom: The higher education institutions in the US, for example, are very good in the research space and not in the innovation space, and some are very good in both spaces. What differentiates the latter institutions? What do they give students so that they can go out with these skills, and are they transferable to other places, such as through the alumni?

Prof Abreu: In your experience in practising innovation did you find special methodologies that would bring more women in?

Response, Dr Comins: I would support the comment that alumni from a university can be a powerful role model. A visit to Stanford showed that 
this idea of co-teaching is used to a great extent. It is about people putting a name and a face and a career path to somebody they can aspire to. When it comes to the point about the need to communicate about women, it would be necessary to get successful women to stand up and be counted and inspire the next generation. In the US and elsewhere, alumni are an integral part of the strategy. I am not sure that we drive this to the same extent in South Africa. Alumni are used for their funding but perhaps less so for their intellect and experience.

Prof Thomson: When I taught biotechnology at the University of Cape Town, I would get alumni whose companies had failed to explain the lessons they had learnt. This was incredibly valuable for the students.

Prof Abreu: What is the mixture of men and women in the Innovation Hub?

Response, Dr Togo: The best way to attract women to the innovation space is to let them do what they do best by providing them with the support and infrastructure they require. One lady who trained as an IT technician now owns a very successful cosmetics company. We need to create an environment that allows women to thrive. We have established satellite innovation hubs in the townships in order to serve the communities and the innovation that comes out of the communities. It is all about helping them to do what they want to do and making them be the best they can.

Prof Buitendijk: Imperial College in London is trying hard to be part of the community. One way is by getting young people in secondary education into our hack spaces and making them aware of entrepreneurial possibilities. We should never forget that education is an integral part of research and innovation. It is important for students to have the right role models. Alumni are, in a way, proxies for society, and universities need to get society into their classrooms, by getting students into partnerships with universities. Their backgrounds and cultural differences can be used in our teaching. This would bring in a view of the future. Outreach early on, using alumni and making teachers more aware of societal global challenges are all ways of tackling this issue. This multifaceted problem needs multifaceted solutions.

Dr Comins: The diagram that I presented was drawn up by an experienced entrepreneur, Prof Olckers. It shows a complexity that each activity is a network in its own right. Partnering and knowing your limitations is a key ingredient. Entrepreneurs learn this the hard way usually. 
Prof Schiebinger: Stanford supports entrepreneurs. The get rich reason for entrepreneurship is not good. Now there is a movement in corporations for social responsibility. We need to bring together social needs and entrepreneurship.

Mr Maphosa: Dr Togo spoke about communicating the innovations and achievements of women. What platforms and mechanisms are available to do this?

Response, Dr Comins: There is a South African Innovation Network (SAIN), which has this as part of its mission. I have been involved in SAIN for a number of years. There are embryo platforms that could be strengthened and this could become a theme that would be very novel. I would be happy to continue this conversation beyond this workshop.

Response, Ms Githuku-Shongwe: UN Women tries to do this across countries. We have a platform called empowerwomen.org where there are stories from across the world about what women are doing, not only about innovation. We are working in partnership to build an innovation platform for women across the world. There may be ways to connect that with SAIN. There has been a big push for UN Women to start looking for new ways of doing things. We have a built a network of innovation champions across the world, and there are conversations about innovation all the time.

Response, Dr Ramsuran: On 15 September, the DST and TIA will be launching an innovation portal for solution seekers and solution providers, and this could also become a portal for people to post their innovation stories.

Prof Abreu: How did ACTS become involved in the gender issue?

Response, Dr Khaemba: ACTS focuses on STI issues, working on different areas of which gender is one. I coordinate the ACTS programme on gender and inclusive development. ACTS has been involved in climate change research since 1992 and has traditionally worked with African governments and groups of negotiators from different areas. This is how we got involved in the policy audit process. We give comments back to government and some of our research is used to inform some of the policies. We have put out work on evidence-based policymaking in Kenya and other places.

Prof Abreu: The International Council for Science (ICSU) used to have a group on climate change and disaster relief. There were some very interesting studies. Community-based women looked out for warning 
signs that were important for prevention. This brought in participation from the local women. Does ACTS have anything like this?

Response, Dr Khaemba: There is an example for Kenya. The Act and the policy recognise evidence from grassroots communities and the like. The Kenyan department that does climate modelling and forecasting called on the community of traditional weather forecasters and they work together to co-create weather information. A radio station shares this information in specific locations. The information helps local communities to know what to do when disasters happen, what they should plant and this enhances food security. ACTS is also engaging in research to see how people with disabilities are taken care of when there is a disaster.

Prof Malcom: What is the extent of collaboration between men and women in other parts of the UN system that is focused on technology, development and the like? The idea of somehow pulling the interests together is important. The CSTD has been overly focused on hightech solutions to the exclusion of things that could be more in line with supporting women's interest in S\&T. The CSTD would probably not have come up with using plastic bags to grow food. Perhaps there is some advantage to putting groups together to better the conditions and situations for both agencies.

Response, Ms Wu: This was the message in the CSTD's May 2017 session. Little bits here and there do not work, particularly if we are to address the ambitious nature of the SDGs. UNCTAD research, for example, focuses on LDCs and we see that the LDCs would have to produce an economic miracle if they are to address the SDGs, particularly if they are to do this in a socially inclusive and sustainable manner. A lot of the SDGs have very strong gender implications. The UN Women's idea of the Buy from Women programme is very inspiring. We are currently looking at renewable energies and want to invite UN Women to talk to us at our panel session in Geneva in November 2017, to showcase some of the locally developed solutions, and sensitise the commissioners who are mostly interested in the newest gadgets.

Prof Abreu: The Rio de Janeiro Research Agency's newsletter published that one of the winners of that year was a young woman who devised a toilet that does not use water but uses microwave technology. This is revolutionary. I do not know if this innovation has ever been commercialised. Implementing ideas on the market is the problem. 


\section{BREAKAWAY SESSIONS}

The breakaway sessions provided the opportunity for participants to have focused discussions on what was heard thus far with the aim of coming up with recommendations at the end of the workshop. The three discussion groups were:

- Group 1: From Research to Applications: Gendered Innovations and Other Challenges, chaired by Dr Comins

- Group 2: Scientific Research and SITE education, chaired by Prof Malcom

- Group 3: Gender Equity in the Attainment of the SDGs: Influencing Policy, chaired by Prof Abreu

Participants participated in a group of their choice.

\section{REPORT TO PLENARY (MODERATOR: IRIS KISJES)}

\section{Group 1: From Research to Applications: Gendered Innovations and Other Challenges (Dr Neville Comins)}

The group devised a goal for gendered innovation: The empowerment of communities through gendered innovation, incorporating the benefits of the woman's lens, and inclusive of men and youth.

ASSAf, as a very reliable developer of evidence-based reporting, should be commissioned to do a study (for advocacy and baseline data gathering) that does an assessment of the current status with respect to the inclusion of gendered innovation in policies and practices, looking at:

- Effectiveness of the current interventions.

- Current practice across government.

- Current key programmes, based on the SDG framework, exploring how they were developed and by whom, and the beneficiaries of these programmes in sectors such as health, transport, water and sanitation, energy and agriculture.

- Indicators.

- Potential partners (C40, UN, Africa Progress Panel).

It was critical to inform government departments, Brazil, Russia, India, China and South Africa (BRICS), the Southern African Development Community (SADC) and the AU that the study was taking place, so that it is not viewed as an investigation. 
The group based the idea of a study on the three categories of 'fixes' (numbers, institutions and the knowledge pipeline) and said that although many policies are in place that mention gender issues, the question is have any of the universities reacted to that in terms of curriculum? Have they taken trends in the policy environment and asked the questions about what the impact on this is on the way the youth is educated? In terms of research, the study should look at the processes used in setting up the research to see if it reflects that aspect, and whether in proposal formats, people are asked about gender issues. Out of the study, it will be necessary to approach the NRF to give guidelines to include this information in research proposals. A baseline study of this nature would allow one to gather crucial evidence and then use the report to start changing attitudes.

Indicators (qualitative and quantitative) would have to be developed with respect to:

- If the beneficiaries of gendered innovation are women, some measure of increased wealth and independence for women and their participation in the economy should be seen. Programmes should measure the wealth and the number of women actors, and ask questions such as:

o How many more women have opened bank accounts?

o Is there an income?

- Are women-owned companies being formed and are they profitable?

- Is there access to the internet (to show whether women can afford the data and so on)?

- Although the Centre for Science and Technology Information (CESTI) does a lot of surveys, their surveys do not cover gendered innovation as OECD parameters are used. It is nevertheless important to have a discussion with them about how to go about measuring some of these indicators and whether they could be added to the CESTI surveys.

- The extent to which planning cycles in various sectors (transport, health, water, energy, agriculture) take gender programmes into account and the progress in this regard.

- Whether institutions have introduced quotas and what their experiences have been (specific gender lens on programmes, values relating to gender parity, access to gender networks and expertise in decision-making). 
The group's discussion about the experience of people in taking research through to the market place came to the conclusion that although it is not without challenges, there is not very much gender bias in the process that exists today. However, there have been instances of challenges encountered by women in research-to-innovation process, such as:

- Gender-bias in the selection of directors of spin-off companies (knowledge-related gender bias).

- All the systems are set up for high-tech innovations and there is limited understanding of incubators. In addition there are many obstacles. Support systems are very fragmented at grassroots level and in indigenous technologies where women are most likely to be innovating.

There is a need to check the innovation pipeline and make sure that it is a coherent pipeline, and make programmatic interventions to address the failures. There is also a need to harmonise institutions along various steps of the pipeline. There should be an overview study to look at the innovation pipeline, not nationally but regionally (Gauteng cannot be used as the benchmark), to identify players, inter-relationships and gaps in each of the regions.

\section{Group 2: Scientific Research and SITE Education (Prof Shirley Malcom)}

The group identified that science research and education has to do with knowledge production that could lead to innovation and knowledge production that has implications in terms of addressing the SDGs in a way that is responsive to a gender lens.

Questions were asked about what science research is, who does it, how, where and for what purpose, and the discussion led to questions about which research comes first - diversity among researchers or within the research? This was a very interesting formulation because if space is made for particular kinds of research, particular kinds of researchers are likely to be attracted. The group realised the importance of targeting funders and influencing the big funding agencies and private donors, and affecting the research agenda and embedded issues such as broader impacts and how to deal with gendered innovations.

The group recommended that funding agencies should be targeted. There is a need to study the implementation of inclusivity/diversity efforts by funders (and learn where it works and where is does not) and to raise questions about special funding programmes focusing on gendered innovations, as well as also the issue of shifting criteria for funding different kinds of programmes. 
Journal publications is another factor that counts in research. There is a need to look at the leadership of the journals, their processes, their review structures and who the reviewers are, and begin to screen for conscious and unconscious bias in order to begin to shift what is valued within the community.

There are some areas of scholarship where gender may be more problematic and where bias can creep in, such as:

- Big data.

- Sequencing (what is chosen to sequence).

- Engineering and design.

- Cheaper innovations and inventions (the issue of being attentive to constraint).

The group recommended that visibility should be given to the bias among/within areas of research, possibly through holding a big meeting with participation from the granting entities, the publishing entities and some of the leading universities. This would contribute to changing the narrative.

There are good examples in biomedical research where people have designed for the South and made it much less expensive. There is a need to gather some of the cases, and look at gender, as well as other intersectionalities within those cases. The group recommended that GenderlnSITE should do a call for case studies to look at gendered innovations from the South, using a template based on Prof Schiebinger's work.

In terms of education, active learning, problem-based learning and the use of maker spaces and technology are highly effective in reaching diverse populations and were opportunities to produce products responsive to the need for gendered innovations. Models that can be transferable should be identified and shared across geographic areas and similar target groups. There may be a need to look at whether the examples, models or structure of the instruction that are provided prevent students from feeling engaged and identifying with the subject area. The group suggested gathering case studies, having discussions regarding culturally responsive teaching and decolonising subject matter.

Issues of classroom space and how it is organised were discussed in the context of good pedagogy and helping students to interact with each other to come up with better ideas. The question of whether 
engineering and computer science students are exposed to thinking with a gender lens and understanding the gender dimension of the things they do is important. This is because these students have low levels of diversity and yet they can have disproportionate impact on innovation and they can build in the bias if they are not attentive to the gender dimension.

The group suggested that:

- Examples of training for gender sensitivity and for innovation, invention and entrepreneurship should be provided.

- A case study of where gender has been considered should be developed in order to help people to start recognising when the difference might occur and what difference this makes.

- TTOs should be catalysts for change, by being on the lookout for gender relevant inventions and targeting them in terms of their own assessment and training that have to do with the gender dimension.

- ASSAf should work with TIA to develop materials to support this component of training around gender-relevant inventions and innovations so that it is standardised across the TTOs.

\section{Group 3: Gender Equity in the Attainment of the SDGs: Influencing Policy (Prof Alice Abreu)}

The group reaffirmed the framework that the SDGs are interconnected to the extent that they cannot be achieved without making the connection between innovation and SDG 5 on gender equality. In addition, the SDGs cannot be achieved without STI and for this the gender lens is essential.

In terms of influencing policymakers, the group felt that policy briefs are important and needed to be more innovative, and looked at some of the ideas used in the Gender Innovations' website, such as a short video aimed at the CEOs of Silicon Valley that could be used to present policy briefs. The group thought about a completely new way of presenting what is important for innovation and gender by using a collective project of this meeting to produce videos of five case studies from five different regions. These would showcase the important factors when talking of gendered innovation, such as:

- A strong gender dimension.

- Social impact and benefits.

- Linked to one of the SDGs. 
- Coming from the South, without dismissing South-North interaction, and different parts of the world.

- Attentive to environmental sustainability and be sustainable.

- Inclusive teams and leadership.

- Implemented (not just an idea).

The group was of the view that there is a wealth of opportunities to implement this project, but would first have to identify sources (institutions that have databases of innovations) and ask for suggestions from the different regions and networks, then list the options and select specific choices of innovations. The next step would be to set up a committee to review the choice of innovations to be showcased and arrange small workshops to discuss the five case studies with their respective innovators. This would be followed by the production of the videos on each of the innovations showing why it is important, how it was produced, the difficulties, and the gender lens it carries. Gender Innovations' network could be used to launch the videos globally. It would stand as an independent project in all the websites in the GenderInSITE network, which would be used to disseminate the videos. The project would have to be presented to funders with the aim of acquiring funding. This big project, although different from the usual report, would have a tremendous impact.

Action items are:

- An analysis of how to influence funders within the context of the funding landscape.

- The incorporation of not only gender equity in the project but also a gender and sex analysis.

Building this project in the different regions would provide a step forward in addressing gender issues. The EU experience, which has very specific guidelines for gender equity and gender analysis, could be looked at and similar incorporations considered.

\section{QUESTIONS AND COMMENTS}

Mr Perkins: What would Group 3 like to happen as a result of the videos?

Response, Prof Abreu: The videos would go to different publics for example, UN Women, UNESCO, UNCTAD and their countries' members and to the GenderlnSITE network. We want them to understand the relevance of a gender lens to innovation. 
Response, Prof Schiebinger: The idea is that we would look at innovations, choose five and make a video of each to describe the project. They would show what the innovation is, how they came to the gender insight, how they are sustainable, how they address the SDG and so on. Each one should be a story in itself and at the end of the videos there would be a lead to everybody's website to get more information and more analysis. We also thought of doing very short written case studies to document this.

Mr Perkins: Do you want funding invested in a particular programme similar to the ones you will be showcasing, or for something else? It is necessary to be clear and strategic about the call to action.

Response, Prof Abreu: The videos and the funding projects are somewhat separate because a more traditional, discussion approach will be used for funders, but the videos could also be shown to the funders to help them understand the importance of having programmes that incorporate a gender lens.

Prof Malcom: A video to funders cannot go without a human being. You need someone to say that this is what we are trying to produce and explain the conditions under which this is possible. Understanding your audience and what they are likely to respond to is absolutely critical.

Mr Perkins: If you are talking to funders, the content needs to include the cost-effective aspect.

Dr Comins: Our group was challenged to look at the entry point and accessing corporates on the subject of gendered innovation. One method is to try and attract some senior captains of industry as champions. A video like this could be a great door-opener to getting CEOs to become ambassadors for this issue.

Prof Diab: It was interesting to see that there was synergy between the groups in terms of case studies. Our group spoke about case studies that have incorporated a gender lens and have been successful (also showing the difficulties experienced on the journey to success) and case studies that have failed and show why they failed.

Mr Perkins: There was more than one call for case studies. It would be interesting to hear the reflections of the three group chairs on whether they would want to go ahead with three different groups of case studies. 
Prof Abreu: The video project would be a collective project of the three sets of outcomes of the three themes discussed, incorporating all the issues raised by the three groups.

Ms Kisjes: Corporate organisations are using certification that gives a stamp of approval to do with the incorporation of gender balance to a certain level. Perhaps this group should do something similar for publishers and research institutions to say they have a gender balance in the way they work and give a certification for this at several levels. This could be a major project.

Prof Malcom: This is an interesting idea. This is starting in the US with regard to universities and colleges. We will be launching this as a way of universities talking about their diversity. In terms of publishers, we will have to think about the criteria needed to get to that point because there are process issues and many things that they could do, but not everything will make a change. Criteria are valuable.

Ms Kisjes: The universities are now looking at diversity and inclusion and not necessarily looking at how they are dealing with unconscious biases or gender analysis in the knowledge process.

Prof Malcom: We have identified areas of overlap and difference. Both have programmatic value. Drilling down in these areas and talking about specific targets for policy action would be useful. It is critical to find champions who can speak to other communities where support is needed. The strategy for getting more voices and a clear message has to be part of the conversation.

Dr Comins: Our group tended to take a local view of the topic. However, it could be that we could help to structure the study being proposed for ASSAf and then it could become a template for some of the other countries in SADC to do something similar, and propagate it in that way.

ProfMalcom: One target should be CSTD because it has members that are from all over the world. There may be some value in informing some of the things that the CSTD does around innovation.

Ms Wu: It would be a natural follow-up to the good work that has been done primarily for the Gender Advisory Board. The videos will bring out the message very clearly and have a huge impact because all the board members are in decision-making positions in their countries. 


\section{DAY 3 (MODERATOR: PROF ALICE ABREU)}

\section{GENERAL DISCUSSION}

\section{Structure of the report (the main issues and how they fit together)}

Some thoughts were shared about a possible structure of the report:

- The first item on the report should be about what innovation is and why the gender lens is needed, bringing in many of the comments from Group 1's discussions and some things that were in the presentations.

- Next, begin to talk about the type of research that is needed.

- Next, talk about why all this is important in relation to the SDGs, making the point about the interconnection of all the SDGs and the importance of having the gender lens and the science.

- Next, a few of the action items (what we still want to know) and the three policy issues (numbers, institutions and the knowledge pipeline). Action points include:

- The case studies project comprising videos and a more specific analysis to substantiate the videos.

- Influencing the funders project.

- A high-level follow-on meeting bringing together publishers, funding agencies and education leaders. They all need to agree that gendered innovation is a challenge that needs to be addressed. Imperial College could be considered as a possible convenor of this meeting.

- Building champions from outside the GenderlnSITE group who can speak to the issues, but are not necessarily from the affected groups.

\section{Clarification of the purpose of the case study project}

The purpose of the case study project is:

- To raise awareness, to motivate and to influence a certain type of public that has links to science and technology policy (i.e. policymakers and funders) about the importance of having a gender lens and there will have to be links to resources as a follow up to the videos.

- To show the value of incorporating gender analysis from the very beginning of any innovation through convening a large number of case studies that represent the complex issue of the links between 
innovation, gender and the SDGs. The SDGs were added to make sure that people understand that implementation of SDGs will need innovation and gender (and science). The case studies should showcase this complex relationship and how gendered innovation has real impact, bringing something new that would not be there without the gendered focus.

To complement a policy brief, there will be five case studies from different regions of the world that illustrate gendered innovation in very different ways depending on the specific cultural settings of the various regions. The influence of context on the case studies will be evident in the videos. Video productions made about the five case studies will highlight the innovation itself and apply to different audiences. In addition, there will be a careful analysis of the case studies that will be used to showcase each study by providing more solid background information and adapted to the different audiences.

\section{Comments}

Participants contributed the following:

- There was a lot of information about bottom-up grassroots innovation with no connection to mainstream research. This should not be lost and should be showcased in the video/case study project because donor funding into developing countries would be strongly influenced by that debate and perhaps it adds a new dimension to their thinking as most of the people affected are probably women and it is driven by women. This is an extremely fertile area to explore from a funding and governmental point of view.

- The relationship between SITE and the social sciences and humanities must be acknowledged, particularly in view of social innovation. This issue should come up in the initial part of the report that talks about what is innovation.

\section{Projects}

- Influencing the funders project:

- The purpose of this project needs to be made clear. (What is funding needed for?)

o It is suggested that Prof Buitendijk and Prof Malcom could be the focal points to suggest how to take this forward and come up with a proposal that is circulated for people to engage with.

- Funders need to be able to respond to whatever strategies are devised. One of the strategies is to fund an honest broker 
who manages the relationships with all the small things, gives out much smaller chunks of money, teaches the people how to manage the budgets, and is responsible for collecting the impact across the groups. This is doable and not as complicated as it seems.

- Case studies project:

- The videos would be the centrepiece of the case study project and an analysis that brings the hard data perspective highlighting relevance to specific audiences should also be presented. This would allow the videos to be useful in a wide audience.

- Videos are a good way to sensitise policymakers and they present the stories that are missing in policy briefs.

- Several stages are envisaged for the project (a network in each region to come up with case studies, a first meeting about the case studies, a meeting with the five selected case studies that were chosen, the video).

- The process of looking for cases studies in each region and choosing five (one from each region) has to be thought through. Some ideas are:

- The identification of the case studies is an important first step. It is necessary to ensure that they map onto the SDGs and to keep the gendered aspects that relate to the SDGs.

- The process should include desktop research as much of the good work being done by women, as well as young scientists and entrepreneurs, is available online, for example on the UN websites.

- Local innovation depends on the characteristics of a specific region or area. If case studies of innovation are to be collected from each region, it would be helpful to have an opportunity to discuss and study the cases to identify the common issues and values. A new collaboration could be developed from the common areas and values identified.

- It is suggested that there is a small co-ordinating group with people from each region (possibly even those not participating in this workshop). The co-ordinating group will have to identify points of entry for regional networks (as opposed to the people who will be gathering to discuss the case studies to be put forward from the region) and therefore should comprise people who understand what the networks are in each region. It is proposed that the following people be part of the co-ordinating group: 
- Africa: ASSAf representative.

- Latin America and GenderlnSITE: Prof Alice Abreu.

- Asia and UNCTAD: Ms Dong Wu.

- Japan: Prof Miyoko Watanabe

- Prof Londa Schiebinger.

- There could be other ways to showcase local case studies that are found as part of the process of looking for the five case studies that will be used in video productions. If ten case studies are found in a specific region, it would be useful to show all ten locally as this would expose work that is being done locally. A collection of case studies would be useful and should be built over time.

- The case study exercise is a collaborative project and would provide the opportunity to cluster local solutions, focusing on impact, and learning from things that look alike so that the collections of case studies will give drivers for moving forward. The collections of case studies in a region will not be lost but will bring a discussion locally that will be picked up by local organisations and used by them in whatever way they find useful.

o The case study project will require funding. A budget would have to be drawn up and funders sought. A big effort would have to be made to acquire the funding by using the expanded networks. GenderlnSITE could provide a little seed money to start the project, (to get people connected, make a list of the databases regionally, distribute the criteria, for example) in order to provide a firm base from which to present a strong project and a strong case to make to a funder.

- It is necessary to have analytical tools (a set of principle questions) that funders ask themselves as they set their goals and make their calls, and case studies to illustrate each one. One way to choose the case studies is to look at what a few key funding agencies are funding in the areas identified, see what the calls are and what they are doing (an audit) in order to be relevant to someone in particular.

- The analysis is crucial to supporting the videos. The analysis was not only about women, but about gender analysis, inclusive analysis. It is important to clarify the role of $S \& T$ in the case studies. This will be done in the analysis of the case studies.

- It would be best to start with a compelling story that can be told to Ministers (they like good stories). It is important to ensure that there is sufficient back-up material once the call to action has been made. 
- The experiences (of Ms Wu and Prof Malcom) with regard to the turning point that made people think about the relevance and the importance of the gender lens and would inform the criteria for the case studies.

- The criteria will have to be agreed on. The first meeting in each of the regions should discuss the criteria and start looking at the databases in the regions for cases that bring the criteria forward. Some thoughts about the criteria are:

- A starting point for developing criteria could be to map some of the examples in the UNCTAD report available online (Applying a Gender Lens to STI (2008)) onto the SDGs. More output and evaluation data about the particular projects and programmes might be available in 2017.

- The case studies must bring together specific correlations about innovation, gender, societal impacts and so on, and bring this paradigm to the discussion.

- The case studies should display rigorous science that is properly referenced so that it is compelling and cannot be dismissed.

- In addition to going about finding case studies, the criteria should be put out to the community to provide case studies. Careful consideration must be given to where the call is put out, and possibly use the Innovation Hub's networks, for example.

- The case studies could reflect whatever works in any form of innovation, and not the 'silver bullet'.

- It would be helpful to start with stringent criteria in order to select the initial five case studies, and relax the criteria over time to make them more inclusive.

- The inclusion of scientific impact in the criteria, although important, would limit case studies of informal, grassroots innovation, which is important for women. However, it would be useful to qualify what is meant by 'sustainability of impact' incorporating the scientific aspect.

- It is necessary to think differently about impact. There is a lot of innovation going on within the informal sector (as shown in the study in Ghana). In the case of using theses case studies to showcase the issues, it would be a missed opportunity not to showcase, for instance, grassroots innovation from the informal sector that might be small but does reflect, at a macro level, an area of significant economic activity. 
- It might be of interest to talk about the enabling policies that help people to innovate, to guide the criteria, and look at what organisations such as UNCTAD have done that has helped empower women. Many of these points will come up in the analysis.

- The CSTD recently did work called, New Innovation Approaches to Facilitate the Implementation of the SDGs. The framing is about the need for innovation in order to achieve the SDGs, and instead of doing more of the same it is important to consider directing innovation towards water, transport, energy systems and so on, and being more inclusive and sustainable.

- Taking a sectoral approach (agriculture, health, infrastructure, energy, water) would be helpful to the World Bank, the African Development Bank, the EU for example, to help them see what they need to change and do differently. This would be about more than advocacy and begin to effect programmatic roll out.

- One understanding of innovation is that it is about changing society to make it better for people. The economic impact and business impact are part of innovation, but innovation is also to enhance the happiness and well-being of people. This important 'blind spot' must be taken into account and was related to a new area that has to do with building capacity for advocacy and the intergenerational nature of this work.

- The following criteria are suggested. Case studies should/must:

- Have a strong gender dimension and a strong influence in bringing out the issue of gender.

- Be innovation with strong social impact (or benefit).

- Be linked to one of the SDGs (reports that address the link between science, SDGs and gender include UNCTAD's report Applying a Gender Lens to STI, the Gender Summit report on SDGs, ICSU report on SDGs).

- Preferably come from the South, although the case study could come from a South-North collaboration.

- Come from different parts of the world - five regions, namely Africa, South Asia, Asia-Pacific, Latin America and the Caribbean, Middle East and North Africa.

- Be cost-effective in terms of implementation.

- Demonstrate sustainability in terms of economic, social, environmental impact (involvement of youth, cultural sensitivity and appropriateness, cost-effectiveness, replication and adaption). 


\section{Other Points Raised about the Projects}

- Clarity was needed about the audience, the tool and the Terms of Reference.

- Careful thought must be given to who the audience of the case study project might be and what funders need to understand about gender in order to be effective with its funding, projects, databases and analyses.

- Audiences that need to be educated about applying the gender lens include panels that review grant applications, as well as the people who write the grant applications (donors and applicants).

- UNCTAD would be very interested in analytical papers and might have funding for this.

- On the new website, there needs to be an explanation of why case studies are being presented, then the higher level abstract, illustrated with the case studies and the narratives.

- Some of the programmes being rolled out by big funding agencies should be looked at. People who understand the issues should be asked to comment on them with the gender lens in mind.

- It was noted that all the case studies could not be hosted on each region's website and that each region should host one case study linking to the other regions in order not to damage search engine optimisation such as Google.

- The two initiatives of the workshop should not be mixed as they will conflate after the videos are ready. However, their work could commence in parallel. The 'influencing the funders group' could start its work. The first meeting with funders could follow this workshop and examples brought about why it is important to have a gender lens. The case study group could commence by looking at what the big funding agencies are funding currently (without a formal audit) and be inspired by those to develop stories around them.

- Gender disaggregated data and other data (statistics) about the situations that people are in are lacking at a global level, particularly to help big funders to know where to invest. The big issues need to be tackled before going down to the narratives.

\section{From Motivation to Implementation}

- It is important to think about the other components to actually make change happen. There is a whole other set of implementation once people are motivated, and there are many implementation-related concerns that have to be addressed. For example, if a funder decides to change the way they do things and apply the gender 
lens their boards will need to be convinced to make the shift. The questions of how they make their cases, do the reviews and work with their staff will have to be addressed. There is much to be done beyond motivating for implementation.

- A multiplicity of organisations is represented at this workshop and this might facilitate the next step, which will address the implementation.

- It might not be necessary to wait to build a whole body of services to support implementation, but use the analysis around the case studies and a de-brief from the analysis as the first generation of a simple tool. Technical outputs would strengthen the videos.

- Once the funders have been influenced and the advocacy videos have been shown, the funding group should look at what the next step should be.

\section{Action Items}

- The video made for Silicon Valley companies on the Gendered Innovations website would be distributed to all participants as an example of the video that would be made from the five case studies (Action: Prof Londa Schiebinger)

- One overall Trellis group with two streams (case studies project and influencing funders project) would be set up with the idea of posting any references (such as studies on the informal sector) that participants think should be looked at. (Action: Ms Erin Johnson/all participants).

\section{MAIN CONCLUSIONS AND RECOMMENDATIONS}

The title and contents page of the report were discussed and participants agreed on the following:

- Title of the report:

The title of the report should introduce more of the SDGs and was suggested as, Gender and Innovation: Implications for Implementation of the Sustainable Development Goals.

- Report contents page and names of individuals who would prepare the draft of each chapter:

- Preface

o Introduction

Purpose of the report (the audience/s, importance of youth awareness)

Explain why this report is focused on innovation (and not S\&T) 
- Chapter 1 (Prof Watanabe)

Relevance to the SDGs, framework of SDGs and interconnectivity of gender with other SDGs. Why is this relevant to the SDGs and why are programmatic interventions needed?

(Allude to the fact that perhaps the SDGs were not correctly formulated and that gender as a cross-cutting issue in SDGs)

- Chapter 2 (Dr Comins, Prof Thomson, Dr Ramsuran, Prof Grobler) What is innovation and why is it important? Why a gender lens? (Contributions from Group 1)

The current landscape of innovation (regional) to contextualise the issue of gendered innovation

Refer to/use Prof Olcker's diagram used in Dr Neville Comins' presentation

- Chapter 3 (Prof Malcom and Ms Wu)

Research and knowledge (S\&T) needed to get the right innovation, including grassroots knowledge (Contribution from Group 2).

Lessons on supporting innovation at grassroots and informal sector (Ghana study)

- Chapter 4 (Prof Abreu, Prof Diab)

Policy and advocacy issues

Highlight instances where gender has not been considered and the implications (economic, but also for happiness and wellbeing).

- Chapter 5 (Prof Abreu, Prof Diab)

Findings, recommendations (further research needed) and action items (2 projects: case studies and influencing funders) Refer to the notes for the group discussions

\section{Other}

- The overall message of the report is that the SDGs will not succeed without gender-inclusive action.

- The overall tone of the report is one of hope and optimism, yet realistic.

- Mr Nick Perkins agreed to be the uber-editor of the report. 


\section{WRAP-UP, CLOSING AND THANKS}

The following actions would be attended to:

- A list of all participants with their contact details would be distributed (Ms Renate Venier).

- The link to the photographs taken of the workshop be sent to all participants (Ms Renate Venier).

- The presentations (including that of Guillermo Anlló) would be shared with all participants (Ms Renate Venier).

It was unfortunate that Guillermo Anllo was unable to attend the workshop. His contribution to the workshop, particularly his important presentation on Why, How and What to measure on Gender Innovation: Many Questions, Few Answers left a gap in the programme.

Prof Abreu expressed her thanks to all the participants on behalf of GenderlnSITE, as well as ASSAf. The variety of organisations represented helped make the workshop particularly interesting and fruitful. The ASSAf team was thanked for their support of the event and Ms Erin Johnson for Tweeting the highlights of the event and for mastering the GenderlnSITE website. 


\section{APPENDIX A: LIST OF ACRONYMS}

AAAS

ACTS

AME

ARUA

ASSAf

AU

BRICS

BRIDGE

CABI

CERRI

CESTI

CEO

CSTD

DFG

DNA

DST

ECOSOC

EDGE

EU

FAPESP

GDP

GenderlnSITE

GGRL

GS10

IAO

ICSU

ICT

IDC

IOT

IP

ISTIC

LDC

LERU

M\&E

MDG

NDP

NGO
American Association for the Advancement of

Science

African Centre for Technology Studies

Artificial Mind Engine

African Research Universities Alliance

Academy of Science of South Africa

African Union

Brazil, Russia, India, China and South Africa

Better Research and Innovation through Diversity and

Gender Equality

Centre for Agriculture and Biosciences International

Centre for Responsible Research and Innovation

Centre for Science and Technology Information

Chief Executive Officer

(UN) Commission on Science and Technology for Development

German Research Funding Organisation

Deoxyribonucleic acid

Department of Science and Technology

(UN) Economic and Social Council

Economic Dividends for Gender Equality

European Union

São Paulo Research Foundation

Gross domestic product

Gender in Science, Innovation, Technology and

Engineering

General Global Research Landscape

Gender Summit 10

Institute for Industrial Engineering

International Council for Science

Information communication technology

Industrial Development Corporation

Internet of Things

Intellectual property

International Science, Technology and Innovation

Centre for South South Co-operation

Least-developed country

League of European Research Universities

Monitoring and evaluation

Millennium Development Goal

National Development Plan

Non-governmental organisation 


$\begin{array}{ll}\text { NRF } & \text { National Research Foundation } \\ \text { NSI } & \text { National System of Innovation } \\ \text { NWU } & \text { North-West University } \\ \text { OECD } & \text { Organisation for Economic Co-operation and } \\ & \text { Development } \\ \text { OWSD } & \text { Organisation for Women in Science for the } \\ & \text { Developing World } \\ \text { PCT } & \text { Patent Co-operation Treaty } \\ \text { PPP } & \text { Purchasing power parity } \\ \text { R\&D } & \text { Research and development } \\ \text { RD\&I } & \text { Research, development and innovation } \\ \text { S\&T } & \text { Science and technology } \\ \text { SADC } & \text { Southern African Development Community } \\ \text { SAGA } & \text { STEM and Gender Advancement } \\ \text { SAIN } & \text { South African Innovation Network } \\ \text { SARChI } & \text { South Africa Research Chair Initiative } \\ \text { SDG } & \text { SUstainable Development Goal } \\ \text { SITE } & \text { Science, innovation, technology and engineering } \\ \text { SMME } & \text { Small, micro and medium enterprise } \\ \text { STEM } & \text { Science, technology, engineering, and mathematics } \\ \text { STI } & \text { Science, technology and innovation } \\ \text { STIP } & \text { Science, Technology and Innovation Policy } \\ \text { STMJ } & \text { Science, Technology and Medical Journals } \\ \text { TIA } & \text { Technology Innovation Agency } \\ \text { TIPC } & \text { Transformative Innovation Policy Consortium } \\ \text { TIOS } & \text { Technology Transfer Offices } \\ \text { TWAS } & \text { The World Academy of Sciences } \\ \text { UK } & \text { United Kingdom } \\ \text { UN } & \text { United Nations } \\ \text { UNCTAD } & \text { UN Conference on Trade and Development } \\ \text { UNESCO } & \text { United Nations Educational, Scientific and Cultural } \\ & \text { Organisation } \\ \text { US } & \text { United States (of America) } \\ \text { VC } & \text { Venture capital } \\ \text { WAITRO } & \text { World Association for Industrial Technology and } \\ \text { WIPO } & \text { Research Organisations } \\ \text { WSIS } & \text { World Intellectual Property Organisation } \\ & \text { World Summit on the Information Society } \\ & \\ & \end{array}$




\section{APPENDIX B: LIST OF PARTICIPANTS}

\begin{tabular}{|c|c|c|c|}
\hline Title & SURNAME & NAME & $\begin{array}{l}\text { INSTITUTION/ } \\
\text { ORGANISATION }\end{array}$ \\
\hline Prof & Abreu & Alice & GenderInSITE \\
\hline Prof & Almeida & Ana Maria & FAPESP \\
\hline Prof & Buitendijk & Simone & Imperial College London \\
\hline $\mathrm{Dr}$ & Comins & Neville & Consultant \\
\hline Prof & Diab & Roseanne & ASSAf \\
\hline Ms & Githuku-Shongwe & Anne & UN Women \\
\hline Prof & Grobler & Anne & North West University \\
\hline Ms & Johnson & Erin & GenderInSITE \\
\hline $\mathrm{Dr}$ & Khaemba & Winnie & ACTS \\
\hline Ms & Kisjes & Iris & Elsevier Foundation \\
\hline $\mathrm{Mr}$ & Krappie & Patrick & TIA \\
\hline Prof & Le Feuvre & $\begin{array}{l}\text { Bruno } \\
\text { (Skype) }\end{array}$ & WIPO \\
\hline $\mathrm{Dr}$ & Maimunah Syed Zin & Sharifah & UNESCO ISTIC \\
\hline Prof & Malcom & Shirley & AAAS \\
\hline $\mathrm{Mr}$ & Maphosa & Stanley & ASSAf \\
\hline Mrs & Marais & Berenice & TIA \\
\hline Ms & Monageng & Kefiloe & TIA \\
\hline Ms & Moonsamy & Senisha & TIA \\
\hline Ms & Nkosi & Nomfundo & ASSAf \\
\hline $\mathrm{Mr}$ & Patel & Imraan & DST \\
\hline $\mathrm{Mr}$ & Perkins & Nick & CABI \\
\hline Ms & Pillay & Sarusha & TIA \\
\hline $\mathrm{Dr}$ & Ramsuran & Anitha & TIA \\
\hline $\mathrm{Dr}$ & Sawers & Jill & FEMTECH \\
\hline Prof & Schiebinger & Londa & Stanford University \\
\hline Prof & Schraudner & $\begin{array}{l}\text { Martina } \\
\text { (Skype) }\end{array}$ & Fraunhofer \\
\hline $\mathrm{Mr}$ & Selepe & Buang & TIA \\
\hline
\end{tabular}


Gender and Innovation: Implications for Sustainable Development

\begin{tabular}{|l|l|l|l|}
\hline \multicolumn{1}{|c|}{ Title } & \multicolumn{1}{|c|}{ SURNAME } & \multicolumn{1}{c|}{ NAME } & \multicolumn{1}{c|}{$\begin{array}{c}\text { INSTITUTION/ } \\
\text { ORGANISATION }\end{array}$} \\
\hline Ms & Sithole & Cynthia & TIA \\
\hline Ms & Somthunzi & Grace & TIA \\
\hline Prof & Thomson & Jennifer & OWSD \\
\hline Dr & Togo & Chamunorwa & Innovation Hub \\
\hline Ms & Venier & Renate & ASSAf \\
\hline Prof & Watanabe & Miyoko & $\begin{array}{l}\text { Japan Science and } \\
\text { Technology Agency }\end{array}$ \\
\hline Ms & Wu & Dong & UNCTAD \\
\hline
\end{tabular}





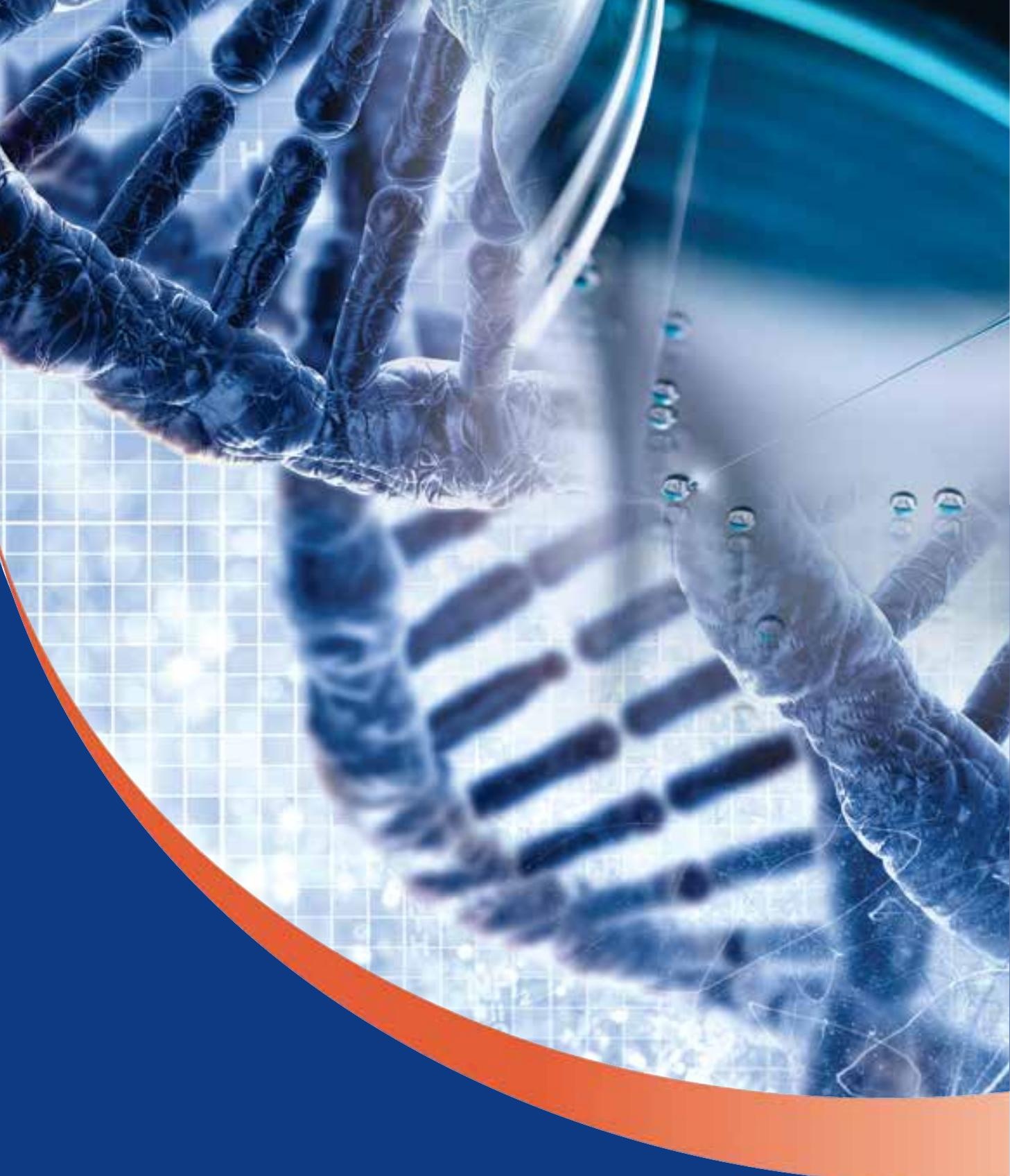

Applying scientific

thinking in the service of society

PO Box 72135, Lynnwood Ridge 0040, Pretoria, South Africa Tel: +27 123496600 • Fax: +27 865769520 • Email: admin@assaf.org.za 


\section{Gender and Innovation: Implications for Sustainable Development}

\section{Academy of Science of South Africa (ASSAf)}

Academy of Science of South Africa (ASSAf)

Academy of Science of South Africa (ASSAf), (2017). Gender and Innovation: Implications for Sustainable Development. [Online] Available at: DOI http://dx.doi.org/10.17159/assaf.2018/0020 http://hdl.handle.net/20.500.11911/98

Downloaded from ASSAf Research Repository, Academy of Science of South Africa (ASSAf) 